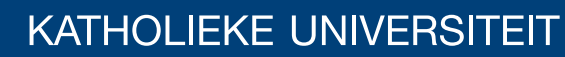 \\ LEUVEN
}

\section{Faculty of Business and Economics}

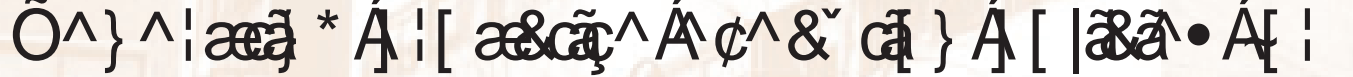

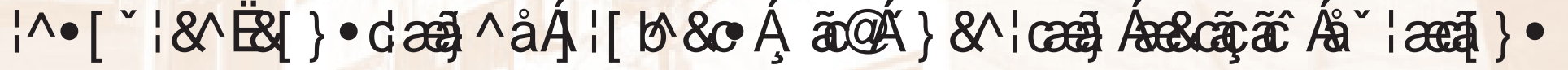

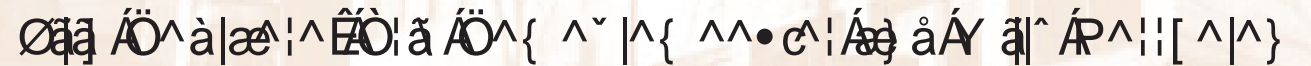

DEPARTMENT OF DECISION SCIENCES AND INFORMATION MANAGEMENT (KBI) 


\title{
Generating proactive execution policies for resource-constrained projects with uncertain activity durations
}

\author{
Filip Deblaere Erik Demeulemeester \\ Willy Herroelen \\ Research Center for Operations Management \\ Department of Decision Sciences and Information Management \\ Faculty of Business and Economics \\ Katholieke Universiteit Leuven (Belgium)
}

February 23, 2010

\begin{abstract}
The resource-constrained project scheduling problem involves the determination of a schedule of the project activities, satisfying the precedence relations and resource constraints while minimizing the project duration. In practice, activity durations may be subject to variability, such that a stochastic approach to the problem is more appropriate. We propose a methodology for the determination of a project execution policy and a vector of predictive activity starting times with the objective of minimizing a cost function that consists of the weighted expected activity starting time deviations and the penalties or bonuses associated with late or early project completion. In a computational experiment, we show that our procedure greatly outperforms existing algorithms described in the literature.
\end{abstract}

\section{Introduction}

Most of the literature in resource-constrained project scheduling involves the determination of a deterministic schedule (with fixed, deterministic activity starting times and activity durations) that can serve as a guideline for the actual execution of the project. During the execution of a project, unexpected events can occur that cause deviations from this schedule. Examples of such events are equipment failure, weather delay, under- or overestimation of the work content, etcetera. The majority of these types of events can be modelled as an activity duration increase or decrease. In Schatteman et al. (2008), for instance, a methodology is proposed for the estimation of the probability and impact of risks on activities of construction projects, and for the generation of an activity duration distribution that incorporates these risks. The so obtained activity duration distributions can then be used to construct a project schedule and/or a project execution policy that takes this activity duration variability into account. 
In the literature, two important research tracks can be identified in the field of project scheduling under uncertainty. A first research track is concerned with the use of a deterministic baseline schedule for the resource-constrained project scheduling problem (or RCPSP) when statistical information about possible disruptions is known. Proactive scheduling procedures can then be used to construct a deterministic schedule that incorporates some protection against possible disruptions (e.g. through time buffering or resource buffering), and reactive scheduling procedures can be invoked during the execution of the project, when disruptions render this schedule infeasible. Typically, the objective of the reactive scheduling procedures is to generate a deterministic reactive schedule that is feasible with respect to the newly available information, and deviates as little as possible from the original baseline schedule. The objective of minimizing deviations from the baseline schedule is sometimes referred to as the stability objective (Van de Vonder, 2006). In the field of proactive/reactive scheduling under the stability objective, the problem of coping with activity duration variability has been tackled in Van de Vonder (2006) and Van de Vonder et al. (2007, 2008). The problem of uncertainty with respect to resource availability has been addressed by Lambrechts et al. (2007, 2008) and Lambrechts (2007). In Yu and Qi (2004), Zhu et al. (2005) and Deblaere et al. (2010), some procedures are presented for reactive scheduling in the multi-mode RCPSP.

A second research track deals with the stochastic resource-constrained project scheduling problem (stochastic RCPSP or SRCPSP), problem $m, 1\left|c p m, \boldsymbol{d}_{j}\right| E\left(C_{\max }\right)$ in the classification of Herroelen et al. (2000). In the SRCPSP, project activities have a known activity duration distribution, and the objective is typically to minimize the expected makespan $E\left(C_{\max }\right)$. Instead of using a deterministic schedule, the execution of a SRCPSP instance is determined through a so-called scheduling policy (Möhring et al. $(1984,1985)$ ). The execution of the project is then treated as a multi-stage decision process where at each decision point the policy acts as a scheduling rule, determining which activities are to be started next. The vast majority of the research in the area of the SRCPSP is concerned with the expected makespan objective only. To the best of our knowledge, no procedures exist that generate SRCPSP policies with some sort of stability objective.

In this paper, we describe a novel proactive project execution approach for singlemode resource-constrained projects with stochastic activity durations. We assume that statistical information is known about the activity durations, and we use this information to generate a dynamic execution policy as well as a vector of predictive activity starting times such that the sum of the weighted expected activity starting time deviations and the (expected) bonuses or penalties associated with early or late project completion are minimized. We show that the proposed approach is both more general and more effective than other state-of-the-art proactive scheduling procedures for the RCPSP with the stability objective described in the literature.

The remainder of this paper is organized as follows. In the next section, we give a formal problem description. Section 3 presents a way to derive the optimal predictive starting times for inflexible activities, given a project execution policy. In Section 4, we describe and motivate our choice to work with the class of resource-based policies with release times. We show how a resource-based policy with release times can be generated that has attractive properties with respect to expected makespan minimization. Section 5 is devoted to our main procedure, in which an initial resource-based policy with release times is optimized for our objective function. The entire solution approach is subsequently illustrated on a simple example in Section 6. In Section 7 we discuss the results of a number of computational experiments and finally, we finish off with some conclusions. 


\section{Problem description}

\subsection{Notation}

Before proceeding to the problem description, we first introduce some notation. We assume that the activity network of a resource-constrained project in activity-on-thenode representation is given by a graph $G(N, A)$ where the nodes $N=\{0,1, \ldots, n+1\}$ represent the activities and the $\operatorname{arcs}(i, j) \in A$ represent the zero-lag finish-start precedence relations. The activities 0 and $n+1$ are the dummy start and the dummy end activity, respectively. We assume the presence of a set of renewable resource types $K$ with a per-period availability $a_{k}, \forall k \in K$. The activities $i$ require a per-period amount $r_{i k}$ of resource type $k$, with $i \in N$ and $k \in K$. We assume that the duration of an activity $i$ is represented by a stochastic variable $\boldsymbol{d}_{i}$ that follows a known distribution. The dummy start and dummy end activity are special cases, as both have a deterministic duration equal to zero.

The execution of a project is performed according to a certain execution policy $\Pi$. Such a policy can be interpreted as a function $\Pi: \mathbb{R}_{n+2}^{+} \rightarrow \mathbb{R}_{n+2}^{+}$that maps a vector $\overrightarrow{\boldsymbol{d}}=$ $\left(\boldsymbol{d}_{0}, \boldsymbol{d}_{1}, \ldots, \boldsymbol{d}_{n+1}\right)$ of activity durations to a vector $\overrightarrow{\boldsymbol{s}}=\left(\boldsymbol{s}_{0}, \boldsymbol{s}_{1}, \ldots, \boldsymbol{s}_{n+1}\right)$ of resource and precedence feasible starting times (Stork, 2001). In the research domain of the SRCPSP, one is interested in finding a policy $\Pi$ that minimizes the expected makespan $E\left(C_{\max }\right)$ of the project, given the probability distributions of the stochastic activity durations $\boldsymbol{d}_{i}$.

\subsection{Problem description}

In the classical SRCPSP, one is mainly interested in finding a policy $\Pi$ that minimizes the expected makespan. For practical applications, however, it is desirable that we are also able to generate a vector of predictive starting times $\overrightarrow{\mathfrak{s}}=\left(\mathfrak{s}_{1}, \mathfrak{s}_{2}, \ldots, \mathfrak{s}_{n}\right)$ that fulfill the role of estimates for the actually realized starting times $\overrightarrow{\boldsymbol{s}}$. These predictive starting times can then serve as a basis for certain coordination purposes involved in the successful execution of the project, such as material procurement, resource planning, fixing agreements with subcontractors, etcetera.

The generation of predictive activity starting times is especially important for socalled "inflexible" activities. Arrangements that have been made based on the predictive starting times of these activities are considered to be difficult or expensive to reschedule. More specifically, if during the execution of the project the realized starting time $\boldsymbol{s}_{i}$ of activity $i$ deviates from its predicted starting time $\mathfrak{s}_{i}$, this will lead to a certain cost. The magnitude of this cost depends on the inflexibility of the activity, the "orientation" of the deviation (positive or negative) and the magnitude of the deviation. For a more formal description, we introduce two additional variable types $\Delta_{i}^{+}$and $\Delta_{i}^{-}$:

$$
\begin{aligned}
\Delta_{i}^{+} & =\left\{\begin{array}{lll}
\boldsymbol{s}_{i}-\mathfrak{s}_{i} & \text { if } & \boldsymbol{s}_{i}>\mathfrak{s}_{i} \\
0 & \text { else } & \\
\Delta_{i}^{-} & =\left\{\begin{array}{ll}
\mathfrak{s}_{i}-\boldsymbol{s}_{i} & \text { if } \\
0 & \text { else }
\end{array} \boldsymbol{s}_{i}<\mathfrak{s}_{i}\right. & i=1, \ldots, n
\end{array}\right.
\end{aligned}
$$

With a positive deviation $\Delta_{i}^{+}$we can now associate a non-negative cost $c_{i}^{+}$per time unit. To give an intuitive explanation for the $\operatorname{cost} c_{i}^{+}$, consider e.g. a construction project. An activity suffering from a positive deviation may for instance require a crane (i.e., an inflexible resource that needs to be reserved in advance) and construction materials. The 
$\operatorname{cost} c_{i}^{+}$could reflect the cost of the prolonged need for the crane as well as additional storage costs for the required materials. With a negative deviation $\Delta_{i}^{-}$(i.e., starting an activity earlier than its predictive starting time $\mathfrak{s}_{i}$ ), we can associate a non-negative cost $c_{i}^{-}$per time unit. These costs are perhaps less important or frequent, but may involve e.g. the costs of acquiring the necessary staff and equipment earlier than planned. Inflexible activities may have $c_{i}^{+}>0$ and/or $c_{i}^{-}>0$. Flexible activities have $c_{i}^{+}=c_{i}^{-}=0$.

For the execution of a project, we assume that a due date $\delta$ is given. With this due date, a nonnegative penalty (e.g. a fine) $p$ can be associated per time unit that the actual project delivery date (or equivalently: the realized starting time of the dummy end activity $\boldsymbol{s}_{n+1}$ ) exceeds the due date. Conversely, a nonnegative bonus $b$ may be awarded per day the project finishes earlier than the due date. Even if there is no explicit bonus incorporated in a given problem setting, early finishes will always result in less fixed costs incurred for keeping the project up and running for an additional day. Let us denote the fixed costs per day by $c_{f}$. Before we can formulate the costs or profits associated with (not) meeting the project due date, we need two additional variables:

$$
\begin{aligned}
& \Delta_{n+1}^{+}=\left\{\begin{array}{lll}
\boldsymbol{s}_{n+1}-\delta & \text { if } \\
0 & \text { else } & \boldsymbol{s}_{n+1}>\delta
\end{array}\right. \\
& \Delta_{n+1}^{-}=\left\{\begin{array}{lll}
\delta-\boldsymbol{s}_{n+1} & \text { if } & \boldsymbol{s}_{n+1}<\delta \\
0 & \text { else }
\end{array}\right.
\end{aligned}
$$

With $\Delta_{n+1}^{+}$we can now associate a cost $c_{n+1}^{+}=c_{f}+p$. Likewise, we have $c_{n+1}^{-}=-c_{f}-b$. Note that $c_{n+1}^{-} \leq 0$ and $c_{n+1}^{+} \geq 0$, such that early finishes are rewarded and late finishes are penalized.

We are now ready to formulate the policy execution cost $\mathcal{C}$ of a policy $\Pi$ and its associated vector of predictive starting times $\overrightarrow{\mathfrak{s}}$ :

$$
\mathcal{C}(\Pi, \overrightarrow{\mathfrak{s}})=\sum_{i=1}^{n+1} E\left(\Delta_{i}^{+} c_{i}^{+}+\Delta_{i}^{-} c_{i}^{-}\right)
$$

This objective function takes into account the costs resulting from positive and negative starting time deviations of the inflexible activities, as well as the fixed project costs, the costs associated with exceeding the project due date and the bonus opportunities related to early project completion. We attract the attention of the reader to the fact that when the project due date is met exactly (i.e., $\boldsymbol{s}_{n+1}=\delta$ ), the component related to the fixed project $\operatorname{costs} c_{f}$ will be equal to zero in the objective function (5). In all other cases, the bonus or penalty related to the fixed costs will (in absolute value) be equal to the fixed costs per time unit $c_{f}$ multiplied by the deviation length. By calculating the fixed costs component relative to the due date instead of relative to the starting time of the project, we are able to consolidate the fixed costs and the explicit penalty or bonus into the single cost factors $c_{n+1}^{+}$and $c_{n+1}^{-}$.

In this paper, we aim to construct a project execution policy $\Pi$ and a vector of predictive starting times $\overrightarrow{\mathfrak{s}}$ that attempt to minimize the policy execution cost (5) for a given resource-constrained project with known activity duration distributions.

\subsection{Robust scheduling and the STC+D heuristic}

The problem as stated in the previous section is related to the topic of robust proactive scheduling addressed in Van de Vonder et al. (2008). In this paper, the same project 
environment is assumed (i.e. resource-constrained projects with stochastic activity durations), and the authors present a number of procedures to generate a buffered deterministic

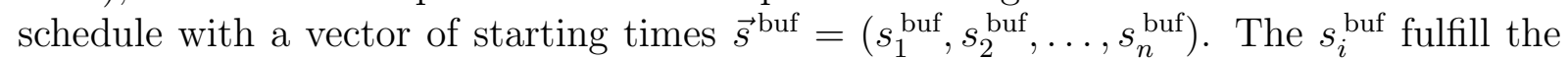
role of predictive starting times, and the schedule $\vec{s}^{\text {buf }}$ is used as a basis for the proposed execution policy $\Pi^{s t c}$ (cfr. infra), that aims to minimize the objective function (6):

$$
\mathcal{C}^{\prime}=\sum_{i=1}^{n} c_{i} E\left|s_{i}^{\text {buf }}-\boldsymbol{s}_{i}\right|+c_{n+1} E\left(\max \left(0,\left(\boldsymbol{s}_{n+1}-\delta\right)\right)\right)
$$

Note that there is only one "symmetric" $\operatorname{cost} c_{i}$ per activity that replaces the asymmetric costs in Equation 5. Furthermore, the authors assume that all $c_{i}$ are nonnegative (in other words, bonuses are not allowed). The buffered schedule consists of activity starting times $s_{i}^{\text {buf }}$ that are resource and precedence feasible with respect to the expected activity durations $E\left(\boldsymbol{d}_{i}\right)$.

The authors propose and evaluate a number of algorithms and conclude that the socalled STC+D heuristic is the preferred solution procedure, yielding very good results in a short average computation time. They also propose a tabu search procedure that yields slightly better results, but the authors mention that the computation time for this latter procedure is too high for large project networks. In the results section, we will compare our procedure with the $\mathrm{STC}+\mathrm{D}$ procedure. We now briefly sketch the logic of the $\mathrm{STC}+\mathrm{D}$ procedure. First, using the expected values $E\left(\boldsymbol{d}_{i}\right)$ as point estimates for the stochastic activity durations $\boldsymbol{d}_{i}$, a starting solution for the basic RCPSP is generated through one of the many (meta)heuristic procedures described in the literature. Then, this schedule is modified by the STC-part of the procedure, that iteratively inserts a single-period time buffer in front of the activity with the highest Starting Time Criticality (STC), while keeping the schedule resource and precedence feasible. This STC value is a surrogate measure for the objective function (6). When the STC procedure has finished, a descent procedure (hence the name "STC+D") further improves the solution by identifying for each activity $i$ a time window $\left[s_{i}^{\min }, s_{i}^{\max }\right]$ where the activity can be started in the current schedule without violating the precedence or resource constraints given the other activity starting times. The best starting time $s_{i}^{*} \in\left[s_{i}^{\min }, s_{i}^{\max }\right]$ is then identified by means of simulation and $s_{i}^{\text {buf }}$ is set equal to $s_{i}^{*}$. The descent continues until no further improvements are found.

The proposed execution policy $\Pi^{s t c}$ is to apply a parallel schedule generation scheme using as a priority list an ordering of activities according to increasing $s_{i}^{\text {buf }}$ value. The authors impose as an additional constraint during schedule execution that $\boldsymbol{s}_{i} \geq s_{i}^{\text {buf }}$. This way, negative starting time deviations and their associated penalties or bonuses can never occur. The authors argue that in some practical contexts, it will be undesirable or even impossible to start activities earlier than planned, because key staff or materials may not be available before the planned starting time. The execution policy $\Pi^{s t c}$ is incorporated in the descent part of the $\mathrm{STC}+\mathrm{D}$ procedure (recall that this part uses simulation) to evaluate possible improving moves. In summary, the $\mathrm{STC}+\mathrm{D}$ procedure delivers a vector of resource and precedence feasible (with respect to the expected activity durations) predictive activity starting times $s_{i}^{\text {buf }}$ and a "matching" execution policy $\Pi^{\text {stc }}$ described above.

The problem description we propose is more general (and more realistic) in that it allows for asymmetric costs as well as early completion bonuses. In the results section, we will compare our procedure with the STC $+\mathrm{D}$ heuristic, the currently best known procedure for this type of problem described in the literature. 


\section{$3 \quad$ Generating optimal predictive starting times}

We are interested in determining an execution policy $\Pi$ and a vector of predictive starting times $\overrightarrow{\mathfrak{s}}$ such that the rescheduling costs (5) are minimized. As will be shown in this section, the problem of obtaining the predictive starting time vector that minimizes (5) can be solved to optimality. For the determination of a suitable execution policy $\Pi$, we will need to resort to heuristics. We discuss the generation of a suitable policy $\Pi$ in Sections 4 and 5.

Given an execution policy $\Pi$, we can easily determine a vector of predictive starting times $\overrightarrow{\mathfrak{s}}$ that minimizes the objective value (5). The steps of this procedure are described in Algorithm 1. For every inflexible activity $i$ we need to determine the value for $\mathfrak{s}_{i}$ that minimizes the policy execution $\operatorname{costs} \mathcal{C}_{i}\left(\Pi, \mathfrak{s}_{i}\right)=E\left(\Delta_{i}^{+} c_{i}^{+}+\Delta_{i}^{-} c_{i}^{-}\right)$with respect to activity $i$. In the special case where the deviation costs are symmetric (i.e. $c_{i}^{+}=c_{i}^{-}$), we simply need to find the median of the stochastic variable $\boldsymbol{s}_{i}$ as it is a well-known result from statistics that the median is the measure of central tendency $\mathfrak{m}$ for a stochastic variable that minimizes the expected absolute deviation of the stochastic variable from $\mathfrak{m}$ (Wasan, 1970).

In the general case where asymmetric costs are possible, we can use the more general formula shown in step 6 of Algorithm 1. The formula presented is nothing else than the classical fractional formula encountered in the newsvendor problem (Arrow et al., 1951). In the newsvendor problem, one wants to predict the optimal volume of newspapers to have in stock given the demand distribution. Per unit that the demand is overestimated, an overage cost will be incurred corresponding to the cost of an unsold newspaper. Per unit that the demand is underestimated, an underage cost will be incurred due to a lost sale. In our problem, we need to find an optimal predictive starting time for each inflexible activity, given its starting time distribution. Per time unit that we overestimate the starting time of the activity $i$, a negative deviation cost $c_{i}^{-}$is incurred. A similar argument can be made for underestimating the activity starting time. Clearly, the formula used in the newsvendor problem can be directly applied to our problem, where the overage costs are set equal to $c_{i}^{-}$and the underage costs are set equal to $c_{i}^{+}$. The use of the logic of the newsvendor problem in scheduling environments with earliness and tardiness costs is not new. In Al-Turki et al. (1996), for instance, a similar formula is used for deriving the optimal due date of a job in the single-machine problem with random job completion times and earliness and tardiness penalties.

For the flexible activities, the value for the predictive starting time $\mathfrak{s}_{i}$ has no influence on the objective function. Therefore, we set it equal to the median of the stochastic variable $\boldsymbol{s}_{i}$. Algorithm 1 uses simulation to determine the vector $\overrightarrow{\mathfrak{s}}$. Since this is a necessary step for the evaluation of the objective function (5), the number of samples of the random vector $\overrightarrow{\boldsymbol{d}}$ required for a sufficiently accurate calculation will be motivated by the resulting error in the objective function. As will be pointed out in Section 5.1, 1000 samples suffice for that purpose.

We note that the computational complexity of the underlying problem forces us to use simulation instead of an analytical evaluation of the objective function. Hagstrom (1988) has shown that in PERT networks, the calculation of the probability of a single point in the distribution of $\boldsymbol{s}_{n+1}$ is $\sharp P$-complete when the activities have two-point distributions. Although this particular result only covers the case of two-point distributions, it is generally assumed that the result can be extended to more complex distributions, which yields the analytical determination of activity starting time distributions very cumber- 


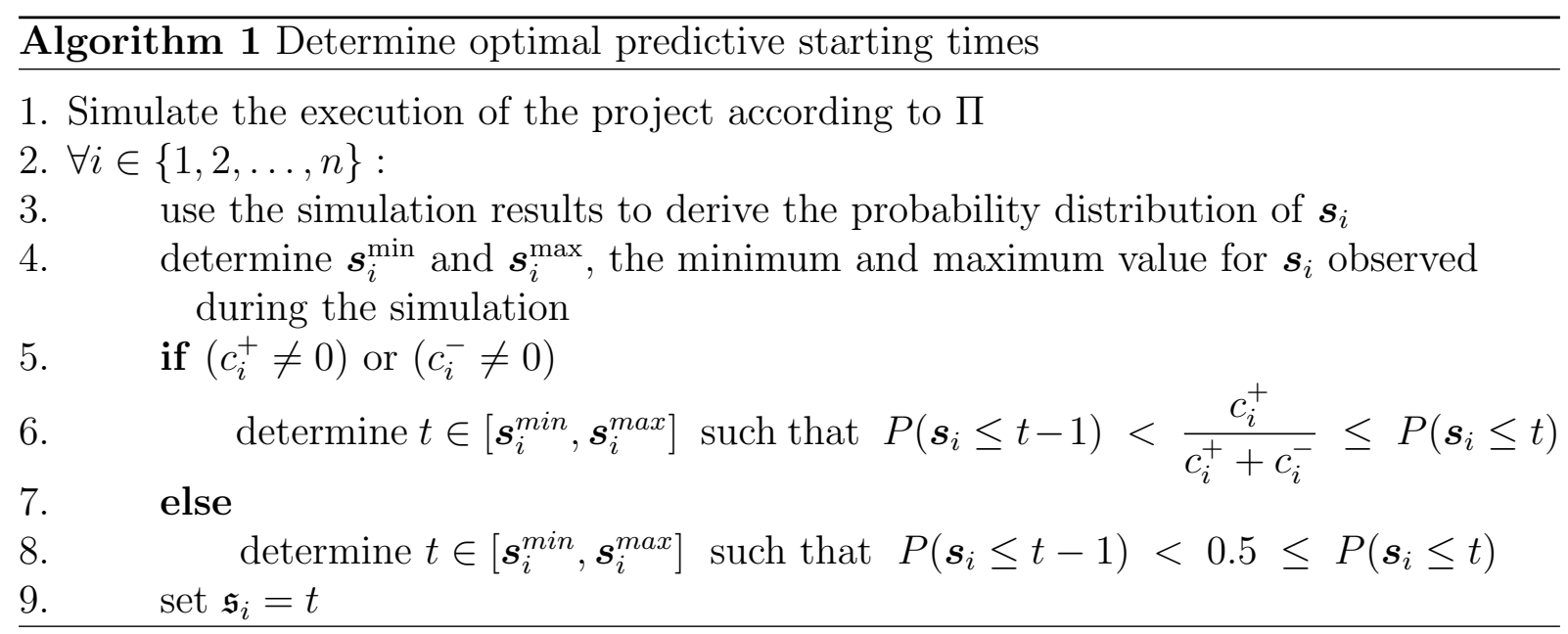

some. Also, in our setting the problem becomes even more complex because the resource constraints need to be taken into account. Therefore, we resort to simulation for the evaluation of the objective function.

\section{Policy class selection}

\subsection{Project execution policies}

As mentioned in the introduction, the execution of a project with stochastic activity durations is done according to a certain execution policy. For our problem setting, we need to select a policy class that is suitable for minimizing the objective function (5). As it seems reasonable to assume that due date performance is an important component of our objective function, it will be of interest to consider a policy class that has good properties with respect to makespan performance, which brings us into the research area of the SRCPSP. Multiple classes of policies for the SRCPSP have been described in the literature. Solution approaches for the SRCPSP typically look for a good (or optimal) execution policy within a certain policy class.

The most important policy classes for the SRCPSP are linear preselective policies, early-start policies, activity-based policies and resource-based policies. The first three policy classes are all subclasses of the more general class of preselective policies. For more information on the class of preselective policies and its subclasses, we refer to Stork (2001). Linear preselective policies and early-start policies are not of interest to us because these policies rely on the determination of all minimal forbidden sets (these are inclusionminimal sets of precedence unrelated activities that can not be scheduled together due to the resource constraints), the number of which can increase exponentially in the number of activities. For problems of realistic size (e.g. 50 activities or more) this is computationally unfeasible, and hence these policy classes will not be considered in further detail.

Activity-based policies use a priority ordering $L$ (respecting the precedence relations) of the project activities. The activities are then started as early as possible and in the order dictated by the list, but never before the starting time of some previously started activity. This implies that the policy introduces a zero-lag start-start precedence relationship between a pair of activities $(i, j)$ whenever $i$ appears before $j$ in the list $L$.

Resource-based policies, also known as priority policies, are a direct extension of the deterministic parallel schedule generation scheme (Brooks and White, 1965). At every de- 
cision time $t$, as many unscheduled activities as possible are started respecting the precedence and the resource constraints. The activities are considered in the order dictated by the list. A resource-based policy is in fact a dynamic version of the parallel schedule generation scheme, because now decision times depend on the realized durations of the nondummy activities, and these durations are only known at the time the corresponding activity finishes. Ashtiani et al. (2009) compare the quality of good resource-based policies to good activity-based policies in terms of expected makespan, and they conclude that resource-based policies yield significantly better results, especially when the activity duration variability is medium to high. This is not very surprising because of the artificially introduced start-start relationships inherent in activity-based policies. Therefore, in this article we will use a policy class that is an extension of the standard resource-based policy class.

\subsection{Resource-based policies with release times}

The policy class we will use in our procedures is the class of resource-based policies with release times. Formally, we will represent a policy $\Pi$ by a pair $\Pi=(\vec{\pi}, \vec{\tau})$ where $\vec{\pi}=\left(\pi_{1}, \pi_{2}, \ldots, \pi_{n}\right)$ represents a vector of priorities and $\vec{\tau}=\left(\tau_{1}, \tau_{2}, \ldots, \tau_{n}\right)$ represents a vector of release times for the nondummy activities. During project execution, at every decision time $t$, a parallel schedule generation scheme will be invoked that uses the priority list implied by the priorities $\pi_{i}$ and only starts a nondummy activity $i$ if the additional condition $t \geq \tau_{i}$ is satisfied. The use of release times $\tau_{i}$ will enable us to control the variability of the starting times of the inflexible activities. For the sake of completeness, we mention that the starting time of the dummy start activity is always equal to zero while the starting time of the dummy end activity always equals the finish time of the latest finishing nondummy activity. As a consequence, for the two dummy activities, no priorities or release times are defined.

Because the class of resource-based policies with release times represents a very large search space, it will be important to find good starting values for the vectors $\vec{\pi}$ and $\vec{\tau}$. We propose to achieve this by solving a deterministic RCPSP instance derived from the resource-constrained project with stochastic activity durations. A multitude of effective heuristic procedures exist nowadays for solving the RCPSP, and we aim to transform a near-optimal schedule for the RCPSP into a resource-based policy with release times for the SRCPSP that performs well on the expected makespan objective. A deterministic RCPSP schedule can be transformed into a policy $\Pi=(\vec{\pi}, \vec{\tau})$ by ordering the activities in the priority list according to increasing starting time, and by using the starting times as release times.

Before we can use this strategy to transform an RCPSP schedule into an SRCPSP policy, we must decide upon a way to translate the activity duration distributions into point estimates for the activity durations. Therefore, we have evaluated five different statistics, namely the mean, the median, the mode, the $40 \%$ percentile and the $60 \%$ percentile. The evaluation of these statistics proceeds as follows. Given an RCPSP instance, a beta distribution is generated for every activity $i$ with an expected value $E\left(\boldsymbol{d}_{i}\right)$ equal to the activity duration, so as to obtain an SRCPSP instance with stochastic activity durations $\boldsymbol{d}_{i}$. Then, for every activity, the point estimate is calculated and a new RCPSP instance is created using these point estimates as activity durations. The modified RCPSP instance is subsequently solved using a heuristic for the RCPSP, and the policy $\Pi$ is constructed from the generated schedule. This policy is then simulated 
(a) Results with release times

\begin{tabular}{|l|c|c|c|}
\hline & Left-skewed & Symmetric & Right-skewed \\
\hline $40 \%$ percentile & $48.30 \%$ & $48.61 \%$ & $48.73 \%$ \\
$50 \%$ percentile & $\mathbf{4 7 . 9 2} \%$ & $\mathbf{4 8 . 3 0} \%$ & $\mathbf{4 8 . 3 7 \%}$ \\
$60 \%$ percentile & $49.77 \%$ & $48.73 \%$ & $48.45 \%$ \\
$E\left(\boldsymbol{d}_{i}\right)$ & $48.14 \%$ & $48.30 \%$ & $48.41 \%$ \\
Mode & $50.55 \%$ & $48.30 \%$ & $48.75 \%$ \\
\hline
\end{tabular}

(b) Results without release times

\begin{tabular}{|l|c|c|c|}
\hline & Left-skewed & Symmetric & Right-skewed \\
\hline $40 \%$ percentile & $\mathbf{4 9 . 6 8 \%}$ & $\mathbf{4 9 . 7 4} \%$ & $49.76 \%$ \\
$50 \%$ percentile & $49.78 \%$ & $49.79 \%$ & $49.84 \%$ \\
$60 \%$ percentile & $49.72 \%$ & $49.78 \%$ & $49.77 \%$ \\
$E\left(\boldsymbol{d}_{i}\right)$ & $49.78 \%$ & $49.79 \%$ & $49.81 \%$ \\
Mode & $49.75 \%$ & $49.79 \%$ & $\mathbf{4 9 . 7 0} \%$ \\
\hline
\end{tabular}

Table 1: Average expected makespan.

on the SRCPSP instance and an expected makespan is obtained. We have tested the different point estimates in three testing circumstances, namely with only left-skewed beta distributions, with only symmetric beta distributions, and with only right-skewed beta distributions.

The right-skewed beta distribution has support $\left[\frac{1}{2} E\left(\boldsymbol{d}_{i}\right), 2 E\left(\boldsymbol{d}_{i}\right)\right]$ and shape parameters 2 and 4 . The symmetric beta distribution has support $\left[\frac{1}{4} E\left(\boldsymbol{d}_{i}\right), \frac{7}{4} E\left(\boldsymbol{d}_{i}\right)\right]$ and shape parameters both equal to $\frac{55}{16}$. The left-skewed beta distribution has support $\left[0, \frac{3}{2} E\left(\boldsymbol{d}_{i}\right)\right]$ and shape parameters 4 and 2 . Note that the width of the support interval is in all three cases equal to $\frac{3}{2} E\left(\boldsymbol{d}_{i}\right)$. We have performed the experiment on the 120 -activity instance set J120 of PSPLIB (Kolisch and Sprecher, 1997). Heuristic schedules for the RCPSP instances have been obtained by the combined crossover algorithm by Debels and Vanhoucke (2006) using 25,000 schedule generations as a stopping criterion. This algorithm is an improved version of the decomposition-based GA described in Debels and Vanhoucke (2007) that has been shown to be among the best performing metaheuristic RCPSP procedures for both small and larger data sets. The expected makespan of the SRCPSP policies has been evaluated by means of simulation using 10,000 scenarios per instance. The simulation has also been performed using an adapted resource-based policy with the same priority vector $\vec{\pi}$ but with $\vec{\tau}$ equal to the zero vector instead of the vector of starting times. We do this to study the impact of release times on the expected makespan, as it might be possible that the release times, that have mainly been introduced to control the activity starting time variability, have a negative impact on the expected makespan.

The results of the experiment are shown in Table 1 . The average expected makespans are reported as the percentage deviation above the critical path length. The critical path length is obtained using the expected values of the corresponding distributions as activity durations. Per column, the best result is highlighted in bold. In Table 1 (a), the results are shown for the policies that use release times. Clearly, the median is the best statistic here. When the activity durations are overestimated (for instance, when using the $60 \%$ percentile), the relatively "late" release times have a negative impact on the expected makespan (this is especially true in the case of left-skewed or symmetric beta distributions). When the activity durations are underestimated (for instance, when 


\begin{tabular}{|l|r|c|c|}
\hline & GRASP & PPGA & $\Pi_{0}$ \\
\hline B1 & $45.60 \%$ & $47.25 \%$ & $47.59 \%$ \\
B2 & $74.17 \%$ & $57.95 \%$ & $59.47 \%$ \\
Exp & $112.37 \%$ & $74.56 \%$ & $76.83 \%$ \\
U1 & $45.21 \%$ & $47.21 \%$ & $47.62 \%$ \\
U2 & $70.95 \%$ & $58.07 \%$ & $61.92 \%$ \\
\hline
\end{tabular}

Table 2: $\Pi_{0}$ compared to state-of-the-art SRCPSP procedures

using the $40 \%$ percentile), the release times will be binding in fewer scenarios, and this too seems to have a negative impact on the expected makespan. This latter conjecture is confirmed when looking at Table 1 (b), where the results without release times are shown. For every column, we see that the best result is always significantly worse than the best result obtained with release times. This is a rather remarkable result, and it implies that it pays off to use release times as an instrument to respect the general structure of the RCPSP schedule from which the policy is derived. This insight has also been confirmed by Tian and Demeulemeester (2010).

Given the results in Table 1 we will choose to use a resource-based policy with release times where the priority list and the release times are derived from an RCPSP schedule with median activity durations. In the remainder of this paper, we will refer to this specific policy as $\Pi_{0}$. The question remains how $\Pi_{0}$ performs when compared to state-ofthe-art procedures for the SRCPSP. To answer this question, we compare our approach to the best procedures around which at the time of writing appear to be the so-called pre-processor policy genetic algorithm (PPGA) described in Ashtiani et al. (2009) and the activity-based policy GRASP proposed by Ballestín and Leus (2009). The former procedure works especially well for the medium and high variability case, while the latter approach gives good results when the variability is low. We have compared our procedure against the GRASP and the PPGA with a schedule limit equal to 25,000. We have used the same continuous activity duration distribution functions as in Ashtiani et al. (2009) and Ballestín and Leus (2009), namely two uniform distributions U1 and U2, one exponential distribution Exp and two beta distributions B1 and B2. The exact description of these distributions can be found in Ashtiani et al. (2009). For the interpretation of our results, it suffices to know that the $\mathrm{U} 1$ and B1 distributions have low variability, the U2 and B2 distributions have medium variability and the Exp distribution has high variability. The results obtained on the J120 instance set, again measured as the average percentage the expected makespan exceeds the critical path length, are shown in Table 2 .

We see that our policy is only slightly worse than the PPGA, and in the medium or high variability case, we obtain much better results than the GRASP algorithm. From these results we may conclude that using a policy $\Pi_{0}$ that is based on an RCPSP schedule is not a bad approach for the SRCPSP at all. The competitive results on the expected makespan objective as well as the ability to control the activity starting time variability are exactly the characteristics we need for an effective minimization of the objective function (5). 


\section{Policy generation}

Now that we have some empirical evidence that $\Pi_{0}$ has good properties with respect to minimizing the expected makespan, we have a starting point for determining a policy that minimizes the objective function (5). In this section, we describe our main procedure that uses a heuristic schedule $\vec{s}=\left(s_{0}, s_{1}, \ldots, s_{n+1}\right)$ (and the policy $\Pi_{0}$ derived thereof) as a starting point to generate a policy $\Pi$ that minimizes (5). The detailed steps of the procedure for generating $\Pi$ and a set of predictive starting times $\overrightarrow{\mathfrak{s}}$ are shown in Algorithm 2. The procedure consists of three parts. First, using a deterministic input schedule, an initial execution policy $\Pi_{1}$ is determined (see Section 5.1) by means of a descent procedure applied to $\Pi_{0}$. We obtain the final policy $\Pi$ by means of an improvement heuristic (see Section 5.2) that uses $\Pi_{1}$ as a starting point, and finally a set of predictive starting times $\overrightarrow{\mathfrak{s}}$ is determined using Algorithm 1.

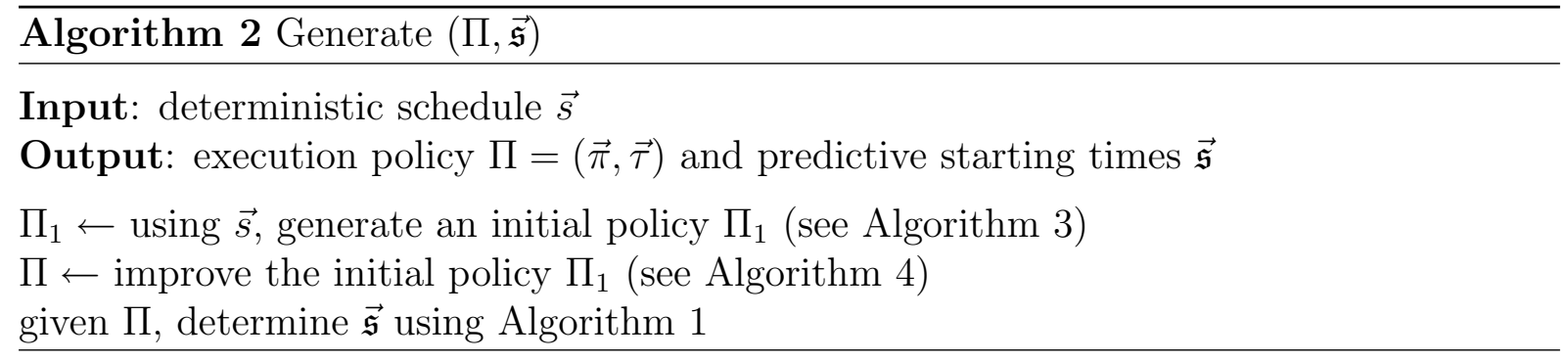

\subsection{Generating an initial policy}

We now proceed to a more detailed discussion of the first two steps of Algorithm 2. In a first step, an initial policy $\Pi_{1}$ is determined using the deterministic input schedule $\vec{s}$. The logic of this procedure is shown in Algorithm 3. Algorithm 3 is essentially a descent procedure that uses the policy $\Pi_{0}$ as a starting point and improves this policy by increasing the release time $\tau_{i}$ of certain activities, thereby reducing their starting time variability. First, the policy $\Pi_{0}$ is determined by setting the release times equal to the starting times in the input schedule, and by ordering the priority list according to "lowest starting time first" where the starting times are again derived from the input schedule. This priority list will not be changed during the execution of Algorithm 3. In Van de Vonder et al. (2007) it is shown that among a large number of priority lists this "lowest starting time first" ordering performs very well in minimizing the expected deviations from the input schedule. We remind the reader of the fact that the input schedule $\vec{s}$ has been obtained by (heuristically) solving an RCPSP instance with median activity durations. In what follows, we will denote $M\left(\boldsymbol{d}_{i}\right)$ as the median of the stochastic variable $\boldsymbol{d}_{i}$.

During the descent procedure, the set of release times $\tau_{i}$ is kept resource and precedence feasible (in the sense that the $\tau_{i}$ would represent a set of feasible activity starting times with respect to the median activity durations $\left.M\left(\boldsymbol{d}_{i}\right)\right)$. We have empirically established that in a descent procedure as described in Algorithm 3, this gives much better results than the alternative (i.e. not imposing any constraints on the $\tau_{i}$ ). A possible explanation for this is that local search procedures are more effective if the initial search space is reduced. Indeed, by restricting the vector $\vec{\tau}$ to the set of feasible starting time vectors, we will be able to move across larger "distances" in the search space, while the unrestricted variant will possibly only observe a very local sample of solution candidates. Also, restricting the initial search space will better conserve the structure of the input schedule $\vec{s}$, which could 
prove beneficial for the expected makespan of the resulting policy. This, in turn, will have a positive effect on the objective function (5).

In order to maintain resource feasibility of the vector $\vec{\tau}$, we use a resource flow network (Artigues et al. (2003), Leus (2003), Leus and Herroelen (2004)). This network reflects the way in which renewable resources are passed on between the various project activities in a given schedule. It has the same set of nodes $(N)$ as the original project network $G(N, A)$, but resource arcs $\left(A_{R}\right)$ are connecting two nodes $i$ and $j$ if there is a strictly positive resource flow $f_{i j k}$ of any resource type $k$ from activity $i$ (when it finishes) to activity $j$ (when it starts). We assume that for every resource type $k$ the sum of all flows out of the dummy start activity equals the sum of all flows into the dummy end activity, both equal to the total resource availability $a_{k}$. Formally:

$$
\sum_{j \in N \backslash\{0\}} f_{0 j k}=\sum_{j \in N \backslash\{n+1\}} f_{j(n+1) k}=a_{k}, \quad \forall k \in K
$$

Moreover, a feasible resource flow network must satisfy the flow conservation constraints at the intermediate nodes. For every resource type $k$ and for every non-dummy activity $i$, the sum of flows into this activity must equal the sum of flows out of this activity, which must be equal to the resource requirement $r_{i k}$. This results in the following constraint:

$$
\sum_{j \in N} f_{i j k}=\sum_{j \in N} f_{j i k}=r_{i k}, \quad \forall i \in N \backslash\{0, n+1\}, \forall k \in K
$$

Finally, a strictly positive flow $f_{i j k}$ can only occur if activity $j$ starts after activity $i$ has finished (recall that the schedule $\vec{s}$ is given):

$$
s_{i}+M\left(\boldsymbol{d}_{i}\right)>s_{j} \quad \Rightarrow \quad f_{i j k}=0, \quad \forall i, j \in N, \forall k \in K
$$

A project network $G(N, A)$ augmented with a set of resource $\operatorname{arcs} A_{R}$ that also act as additional precedence relations, has the property that any early start schedule is resource feasible, regardless of the actual activity durations $\boldsymbol{d}_{i}$ that may differ from their median value $M\left(\boldsymbol{d}_{i}\right)$. In other words, there are no forbidden sets in the project network $G^{+}(N, A \cup$ $\left.A_{R}\right)$. For a given schedule $\vec{s}$, there may be different resource flow networks satisfying Equations (7-9). In Algorithm 3, we use the single-pass algorithm by Artigues et al. (2003) to generate a resource flow network.

In steps 5, 11 and 17 of Algorithm 3, the objective function (5) must be evaluated. This is done through simulation using 1000 samples of the random vector $\overrightarrow{\boldsymbol{d}}$. We found that, in 120-activity networks, a sample size of 1000 results in an average relative error of $0.95 \%$, which is sufficiently accurate for optimization purposes. Note that the evaluation of Equation (5) requires us to use Algorithm 1 to determine the optimal $\overrightarrow{\mathfrak{s}}$ vector.

In Algorithm 3, a list $\mathcal{L}$ is maintained of nondummy activities that are candidates for having their release time $\tau_{i}$ increased. The activity $i \in \mathcal{L}$ with the highest contribution to the objective function (5) will be considered first. We increase its release time (step 12) and the release times of its successors in the network $G^{+}\left(N, A \cup A_{R}\right)$, if necessary (steps 13-16). This new vector $\vec{\tau}$ is evaluated and if it results in a decrease of the objective function, we keep the new vector of release times. If this is not the case, we restore the vector $\vec{\tau}$ as saved before the move, we remove the activity from the list and continue the search for an improving move in the reduced activity list $\mathcal{L}$. Removing activities from consideration during the while-loop of step 10 not only results in a speed increase, it also results in better solutions with respect to the objective function (5). Apparently, moves 
Algorithm 3 Generate initial policy $\Pi_{1}$

Input: deterministic schedule $\vec{s}=\left(s_{0}, s_{1}, \ldots, s_{n+1}\right)$

Output: execution policy $\Pi_{1}=(\vec{\pi}, \vec{\tau})$

1. Determine $\Pi_{0}$ as follows:

$\forall i \in N \backslash\{0, n+1\}$, set $\tau_{i}=s_{i}$

sort the activities $i \in N \backslash\{0, n+1\}$ according to nondecreasing $s_{i}$; this yields

an activity list $\vec{a}=\left(a_{1}, a_{2}, \ldots, a_{n}\right)$; set $\pi_{a_{j}}=j, \forall j \in N \backslash\{0, n+1\}$ set $\Pi_{0}=(\vec{\pi}, \vec{\tau})$

2. $\Pi_{1} \leftarrow \Pi_{0}$

3. generate a resource flow network $G^{\prime}\left(N, A_{R}\right)$

4. generate a topological ordering $\left(l_{1}, l_{2}, \ldots, l_{n}\right)$ of the activities $i \in N \backslash\{0, n+1\}$ such that $l_{i}<l_{j} \forall\left(l_{i}, l_{j}\right) \in\left(A \cup A_{R}\right)$

5. $C \leftarrow \mathcal{C}\left(\Pi_{1}, \overrightarrow{\mathfrak{s}}\right)$

6. repeat:

7. $\quad C_{\text {old }} \leftarrow C$

8. $\mathcal{L} \leftarrow N \backslash\{0, n+1\}$

9. $\quad$ save $\vec{\tau}$

10. $\quad$ while $\mathcal{L} \neq \emptyset$

11. $\quad i \leftarrow \arg \max \left(\mathcal{C}_{i}\left(\Pi_{1}, \mathfrak{s}_{i}\right)\right)$

12. $\tau_{i} \leftarrow \tau_{i}^{i \in \mathcal{L}}+1$

13. find the index $m$ such that $l_{m}=i$

14. for $j=l_{m+1}, l_{m+2}, \ldots, l_{n}$ :

15. $\quad$ if $(i, j) \in\left(A \cup A_{R}\right)$ and $\tau_{i}+d_{i}>\tau_{j}$ :

16. $\quad \tau_{j} \leftarrow \tau_{i}+d_{i}$

17. $\quad C^{\prime} \leftarrow \mathcal{C}\left(\Pi_{1}, \overrightarrow{\mathfrak{s}}\right)$

18. if $C^{\prime}<C$ :

19. $\quad$ set $C \leftarrow C^{\prime}$ and go to step 9

$20 . \quad$ else

21. $\quad$ restore $\vec{\tau}$, set $\mathcal{L} \leftarrow \mathcal{L} \backslash\{i\}$

22. until $C_{\text {old }}=C$ 
that have been rejected once are not the best candidates to reconsider in the near future. Only if the list $\mathcal{L}$ is empty and improving moves were made, we revoke the "tabu" status of all activities and we restart the search for improving moves. Doing so ensures that we finish in a local optimum w.r.t. the proposed neighborhood.

\subsection{Improving the initial policy}

In the second step of our policy generation procedure (see Algorithm 2), we subject the initial policy $\Pi_{1}$ to an improvement procedure. The main differences between the improvement procedure and the initial policy generation procedure are that we now remove the precedence and resource feasibility constraints on the vector $\vec{\tau}$ and that we allow the priority vector $\vec{\pi}$ to change. The improvement procedure is outlined in Algorithm 4. It consists of three major steps that are repeated as long as one of the steps yields an improvement. We now proceed to a more detailed discussion of each of the three steps.

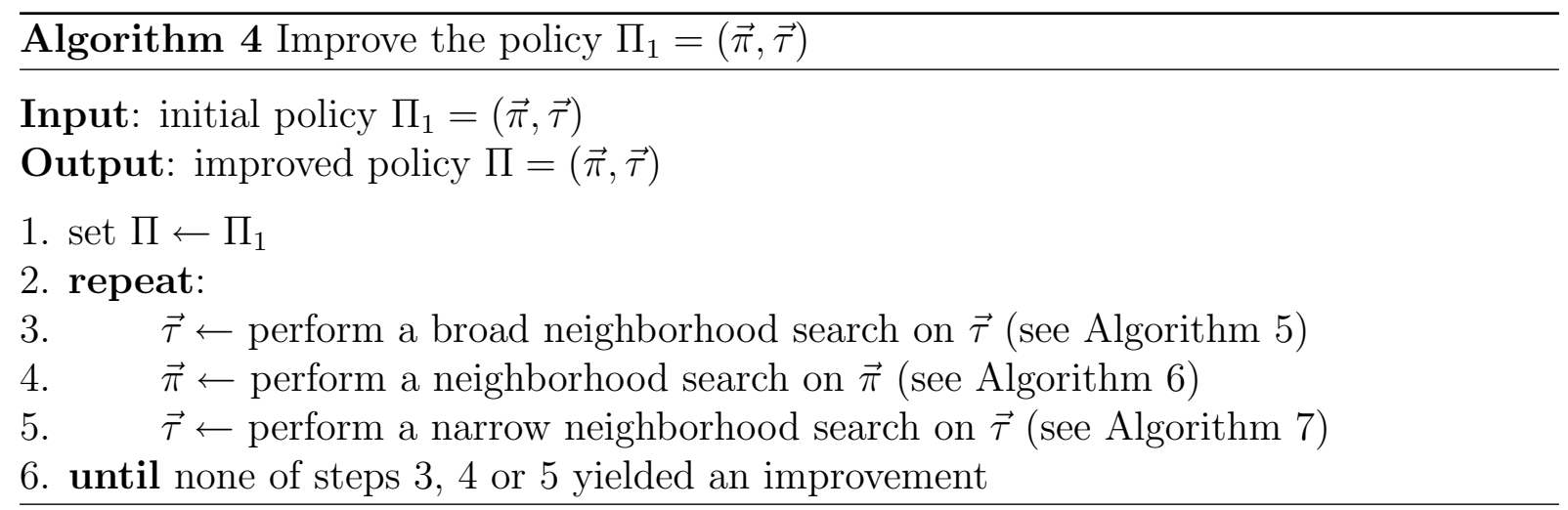

\subsubsection{Broad neighborhood search on $\vec{\tau}$}

In a first step, we perform a broad neighborhood search on the release time vector $\vec{\tau}$. Before we can proceed to the formal description of the procedure, we need some additional notation. We denote $\boldsymbol{d}_{i}^{\text {min }}$ as the minimum duration of activity $i$, i.e. the minimal value for the stochastic variable $\boldsymbol{d}_{i}$ with a strictly positive probability of occurrence. The symbol $\boldsymbol{s}_{i}^{\text {min }}$ has the same meaning as in Algorithm 1 . Finally, $L P L(i, j)$ denotes the length of the longest path between activities $i$ and $j$ (inclusive) in the network $G(N, A)$ with respect to the minimal durations $\boldsymbol{d}_{i}^{\text {min }}$. The detailed steps of the broad neighborhood search procedure are shown in Algorithm 5.

As was the case in Algorithm 3, a list $\mathcal{L}$ of nondummy activities is maintained and the activity $j \in \mathcal{L}$ with the highest contribution to the objective function is considered first. For an activity $j$, we consider all alternative release times except for the "nearest" alternatives $\tau_{j}-1$ and $\tau_{j}+1$, because these cases are treated by the narrow descent procedure (cfr. infra). Considered activities are always removed from the list since for a given activity (almost) all values for $\tau_{j}$ that could lead to an improvement of the objective function are evaluated. Therefore, a recently considered activity is not likely to lead to another improvement in the first subsequent steps of the procedure. Alternative candidate release times $\tau_{j}$ are evaluated through simulation using a small number of scenarios (namely 100), hence the symbol $\mathcal{C}^{\text {fast }}$. We keep track of the best new objective value $C_{\text {min }}$ with respect to this approximate evaluation of Equation 5 and the corresponding release time $t^{*}$. Due to the small number of scenarios, there is no guarantee that $t^{*}$ will be the 


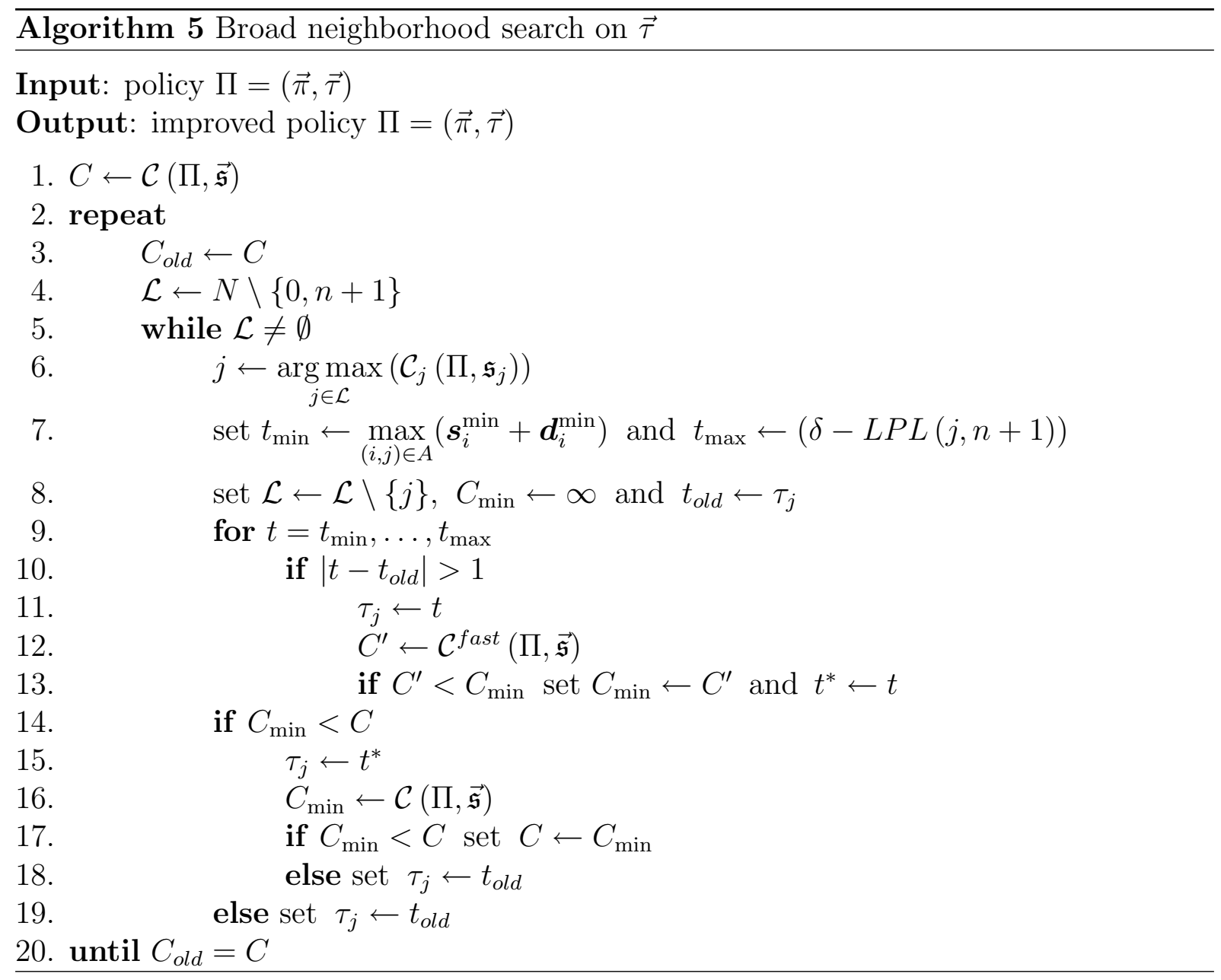


best new value for $\tau_{j}$, but at the least it will be one of the better candidate release times. When $t^{*}$ has been identified, we check whether the corresponding objective value $C_{\text {min }}$ is indeed an improvement compared to the previous objective value $C$ (step 14). Note that this decision can also be the subject of error. To eradicate such "false positives", we perform an accurate evaluation of the objective function through 1000 simulation steps (step 16) and we accept the move if there is indeed an improvement. In the other case, $\tau_{j}$ is restored to its former value. If the list $\mathcal{L}$ is empty and the while loop of step 5 resulted in an improvement, the list is refilled and the improvement loop restarts.

\subsubsection{Neighborhood search on $\vec{\pi}$}

The second step of the improvement algorithm (see Algorithm 4) is concerned with improving the priority vector $\vec{\pi}$. The detailed steps of this procedure are shown in Algorithm 6 . Recall that $\pi_{i}$ represents the priority of activity $i$ and we have $\pi_{i} \in\{1, \ldots, n\}$. For $i \neq j$ we have $\pi_{i} \neq \pi_{j}$, such that the vector $\vec{\pi}$ is actually a permutation of the set $\{1, \ldots, n\}$. If during the execution of the policy $\Pi$ two activities $i_{1}$ and $i_{2}$ become eligible, the activity with the highest $\pi_{i}$ value will be started first. The procedure shown in Algorithm 6 iterates over all nondummy activities $i$ and verifies whether priority decreases or increases of an activity yield better results with respect to the objective function (5). The working logic of Algorithm 6 is best explained through a small example. Suppose we have $n=7$ and a priority vector $\vec{\pi}=(3,5,4,7,6,1,2)$. Translating this vector into a priority list where the activities are ordered according to decreasing priority yields the list $l(\vec{\pi})=(4,5,2,3,1,7,6)$. If during Algorithm $6, i=3$, we will be looking at the effect of increasing and decreasing the priority of activity 3 . Increasing the priority will result in the evaluation of the lists $l=(4,5, \mathbf{3}, 2,1,7,6), l=(4, \mathbf{3}, 5,2,1,7,6)$ and $l=(\mathbf{3}, 4,5,2,1,7,6)$. Decreasing the priority will result in the evaluation of the lists $l=(4,5,2,1, \mathbf{3}, 7,6)$, $l=(4,5,2,1,7, \mathbf{3}, 6)$ and $l=(4,5,2,1,7,6,3)$.

In step 10 of Algorithm $6, T(A)$ denotes the transitive closure of the arc set $A$, defined as $T(A)=\{(i, j) \mid$ there is a path from $i$ to $j$ in the directed graph $A\}$. We will call a priority increase or decrease of an activity $i$ effective if the relative ordering of activity $i$ changes in relation to some activity $j$ with $(\{(i, j),(j, i)\} \cap T(A))=\emptyset$. Only effective priority changes can give rise to a difference in the objective value. Indeed, if $i$ and $j$ are precedence related, we need not evaluate the new priority list as the precedence relation will always be respected regardless of the priority values $\pi_{i}$ and $\pi_{j}$. For a given activity, we will consider $k_{\max }$ effective priority decreases and $k_{\max }-1$ effective priority increases. We may consider one less increase because the first effective increase of $\pi_{i}$ is equivalent to the first effective decrease of the priority of some activity $j$ with $\pi_{j}>\pi_{i}$. Translated to the lists example, we do not need to evaluate the priority increase $l=(4,5,3,2,1,7,6)$ of activity 3 if the priority decrease $l=(4,5,3,2,1,7,6)$ of activity 2 has been evaluated earlier. Steps 17 through 20 of Algorithm 6 result in skipping the first effective priority increase.

Algorithm 6 consists of two analogous parts. Steps 5 through 13 are concerned with decreases of $\pi_{i}$ while steps 14 through 27 are concerned with increases of $\pi_{i}$. For a given activity, the first improving priority change will always be accepted, after which the search proceeds with the next activity. The procedure terminates when a full scan of the activity list does not result in an improving move. 


\section{Algorithm 6 Neighborhood search on $\vec{\pi}$}

Input: policy $\Pi=(\vec{\pi}, \vec{\tau})$

Output: improved policy $\Pi=(\vec{\pi}, \vec{\tau})$

1. $C \leftarrow \mathcal{C}(\Pi, \overrightarrow{\mathfrak{s}})$

2. repeat

3. $\quad$ set $C_{\text {old }} \leftarrow C$ and $i \leftarrow 1$

4. $\quad$ while $i \leq n$

5. $\quad k \leftarrow 0$

6. $\quad$ save $\vec{\pi}$

7. $\quad$ while $\left(k \neq k_{\max }\right)$ and $\left(\pi_{i}>1\right)$

8. $\quad$ find $j \in N \backslash\{0, n+1\}$ with $\pi_{j}=\left(\pi_{i}-1\right)$

9. $\quad$ set $\pi_{j} \leftarrow\left(\pi_{j}+1\right)$ and $\pi_{i} \leftarrow\left(\pi_{i}-1\right)$

10. $\quad$ if $(\{(i, j),(j, i)\} \cap T(A))=\emptyset$

11. $\quad C^{\prime} \leftarrow \mathcal{C}(\Pi, \overrightarrow{\mathfrak{s}})$

12.

13.

14.

15.

16.

17.

18.

19.

if $C^{\prime}<C$ set $C \leftarrow C^{\prime}, i \leftarrow(i+1)$ and go to step 4

(3.

if $k_{\max } \neq 1$

else set $k \leftarrow(k+1)$

20.

21.

22.

23.

24.

25.

26.

27.

28.

$$
k \leftarrow 0
$$

restore $\vec{\pi}$

while $(k \neq 1)$ and $\left(\pi_{i}<n\right)$

find $j \in N \backslash\{0, n+1\}$ with $\pi_{j}=\left(\pi_{i}+1\right)$

set $\pi_{j} \leftarrow\left(\pi_{j}-1\right)$ and $\pi_{i} \leftarrow\left(\pi_{i}+1\right)$

if $(\{(i, j),(j, i)\} \cap T(A))=\emptyset$ set $k \leftarrow(k+1)$

while $\left(k \neq k_{\max }\right)$ and $\left(\pi_{i}<n\right)$

find $j \in N \backslash\{0, n+1\}$ with $\pi_{j}=\left(\pi_{i}+1\right)$

set $\pi_{j} \leftarrow\left(\pi_{j}-1\right)$ and $\pi_{i} \leftarrow\left(\pi_{i}+1\right)$

if $(\{(i, j),(j, i)\} \cap T(A))=\emptyset$

$C^{\prime} \leftarrow \mathcal{C}(\Pi, \overrightarrow{\mathfrak{s}})$

if $C^{\prime}<C$ set $C \leftarrow C^{\prime}, i \leftarrow(i+1)$ and

go to step 4

29.

else set $k \leftarrow(k+1)$

30. until $C_{\text {old }}=C$ 


\subsubsection{Narrow neighborhood search on $\vec{\tau}$}

In the final step of the improvement procedure (see Algorithm 4), we perform a narrow neighborhood search on the release time vector. The detailed steps of this straightforward procedure are presented in Algorithm 7. The list $\mathcal{L}$ contains, as before, the inflexible nondummy activities. Activities in this list are considered in the order of "largest contribution to the objective function first". For an activity $i$, we consider the alternative release times $\tau_{i}+1$ and $\tau_{i}-1$. These two alternatives are evaluated using simulation and an improving move is immediately executed. Once a move has been made, we restart the search for an improving move with a full list $\mathcal{L}$. The search terminates as soon as no activity in $\mathcal{L}$ has an improving move. The use of this procedure guarantees that we finish in a local optimum with respect to the narrow neighborhood.

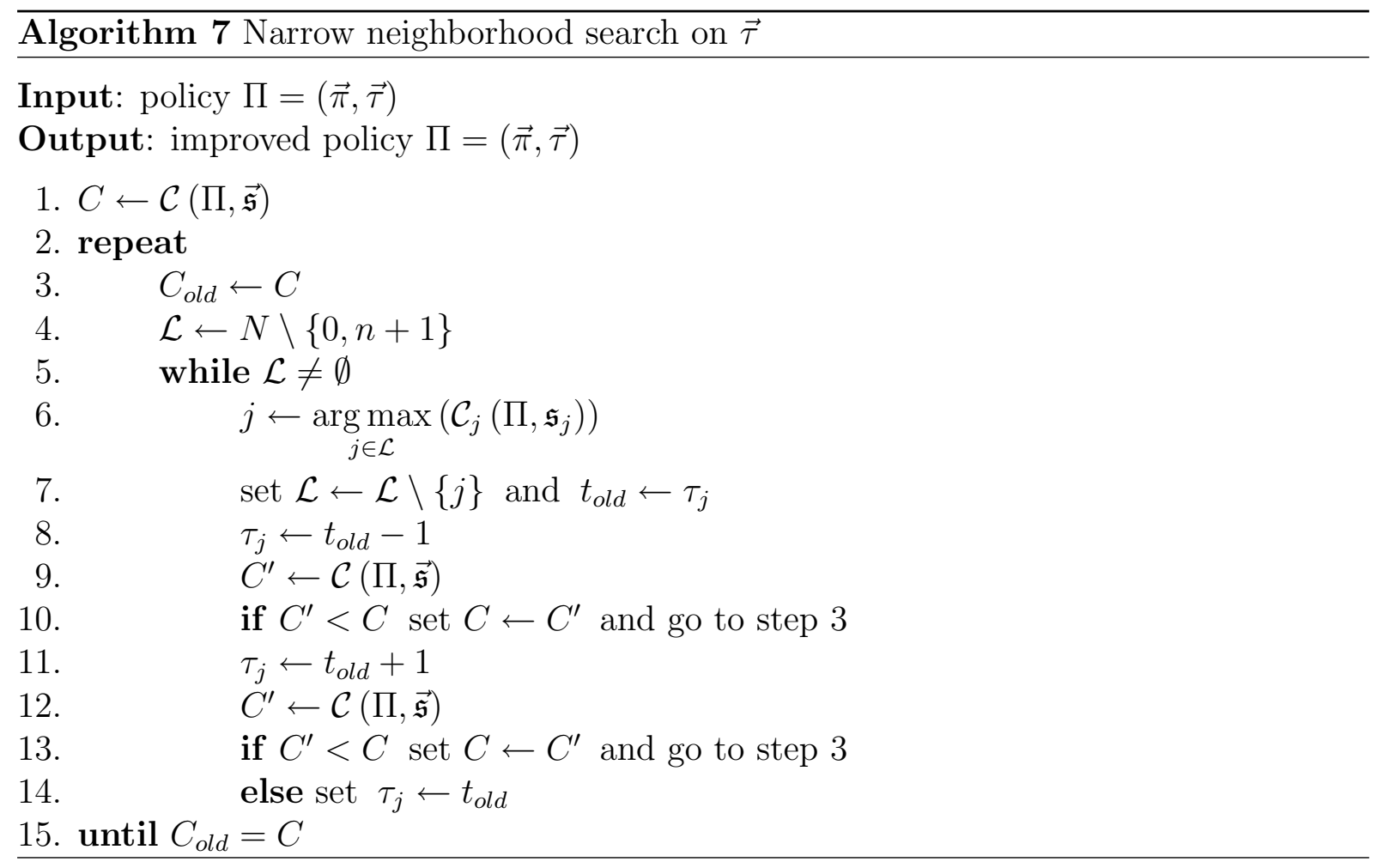

\section{Example}

To show some of the benefits of Algorithm 2, we illustrate it on an example instance. In Figure 1, a project network along with a makespan minimizing RCPSP schedule is shown. Every nondummy activity $i$ is equipped with a stochastic activity duration $\boldsymbol{d}_{i}$ that follows a discretized beta distribution. The characteristics of this distribution will be described in Section 7. The nondummy activities in this particular example all have a median duration $M\left(\boldsymbol{d}_{i}\right)$ equal to the expected duration $E\left(\boldsymbol{d}_{i}\right)$. In the left pane, the activity number is shown inside the node, the expected duration $E\left(\boldsymbol{d}_{i}\right)$ is shown above the node and the requirement $r_{i 1}$ for the single renewable resource type with per-period availability $a_{1}=12$ is shown below the node. In the right pane, the resource profile corresponding to a makespan minimizing RCPSP schedule is shown. The resource utilization is shown on the $Y$-axis and the time is indicated on the $X$-axis. A due date $\delta=15$ is imposed on the project. The per-period penalty of exceeding this due date equals $c_{7}^{+}=20$. There is no 


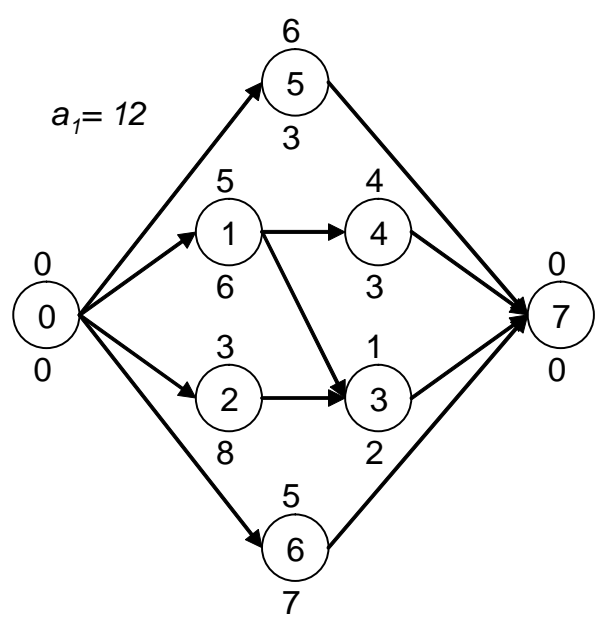

(a) Project network

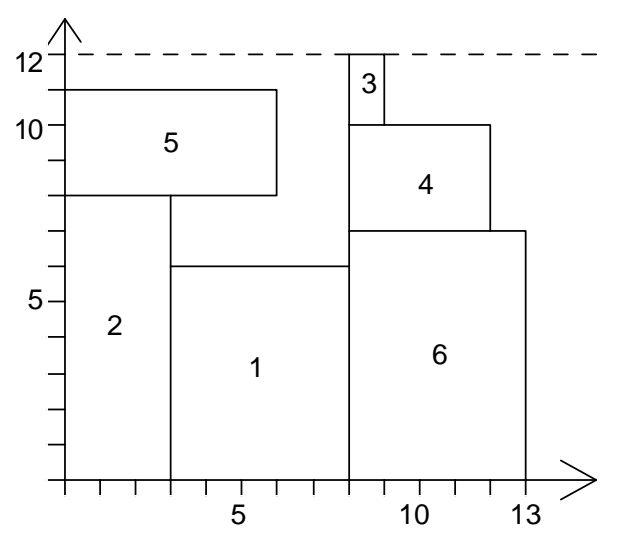

(b) Makespan minimizing schedule

Figure 1: Example instance

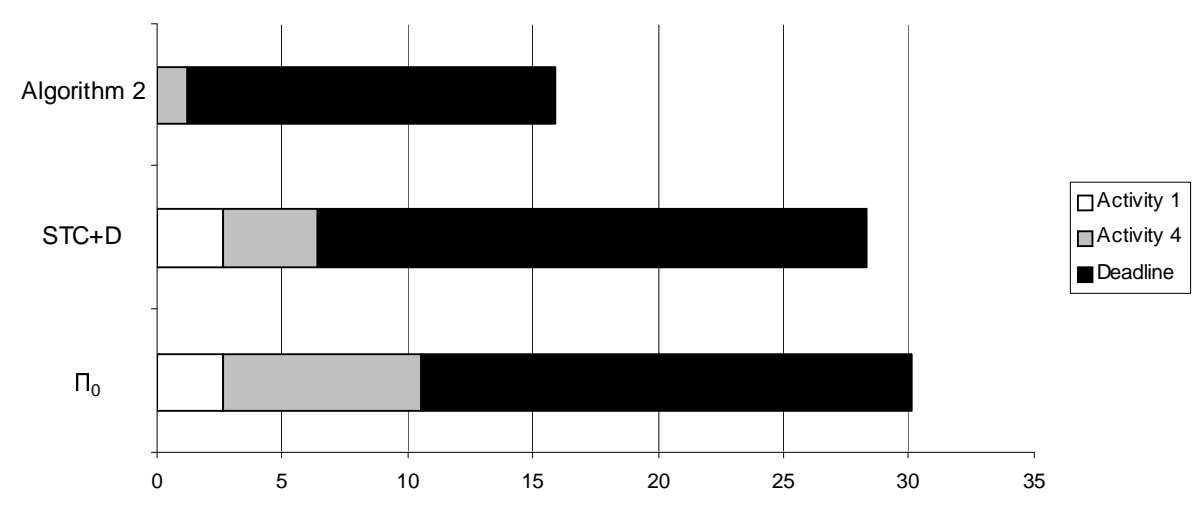

Figure 2: Policy execution costs

earliness bonus. There are two inflexible activities, namely activities 1 and 4 for which we have $\left(c_{1}^{-}, c_{1}^{+}\right)=(2,7)$ and $\left(c_{4}^{-}, c_{4}^{+}\right)=(4,8)$.

We simulate the execution of the project according to three different policies. The first policy is the policy $\Pi_{0}$. In this case (where we have $M\left(\boldsymbol{d}_{i}\right)=E\left(\boldsymbol{d}_{i}\right), \forall i$ ), it is the starting point of both the STC+D procedure and Algorithm 2, and it can be thought of as the "default" policy when one wants to execute a deterministic schedule like the one shown in Figure 1 (b) without further optimization of the objective function (5). Figure 2 shows a breakdown (with respect to the source of the costs) of the objective function value for the $\Pi_{0}$ policy, the STC $+\mathrm{D}$ policy and the Algorithm 2 policy. Both $\mathrm{STC}+\mathrm{D}$ and Algorithm 2 succeed in improving the initial policy $\Pi_{0}$, but Algorithm 2 does a much better job than the STC+D procedure. In order to understand what has happened, we need to take a closer look at the behavior of the three different policies.

In Figure 3, we show a graphical illustration of the different policies in what we call a stochastic Gantt chart. This concept was first introduced by Baker and Trietsch (2009) who refer to it as a predictive Gantt chart. Like in a regular Gantt chart, the execution intervals of the activities are indicated by a horizontal bar per activity. A detail of the stochastic Gantt chart of Figure 3 (a) is displayed in Figure 4. Figure 4 corresponds to the fourth horizontal bar shown in Figure 3 (a). The solid line and the dashed line represent the cumulative probability distribution of the start time and the finish time of activity 4 , respectively. The left edge of the grey bars in Figure 3 represents the minimum value for 


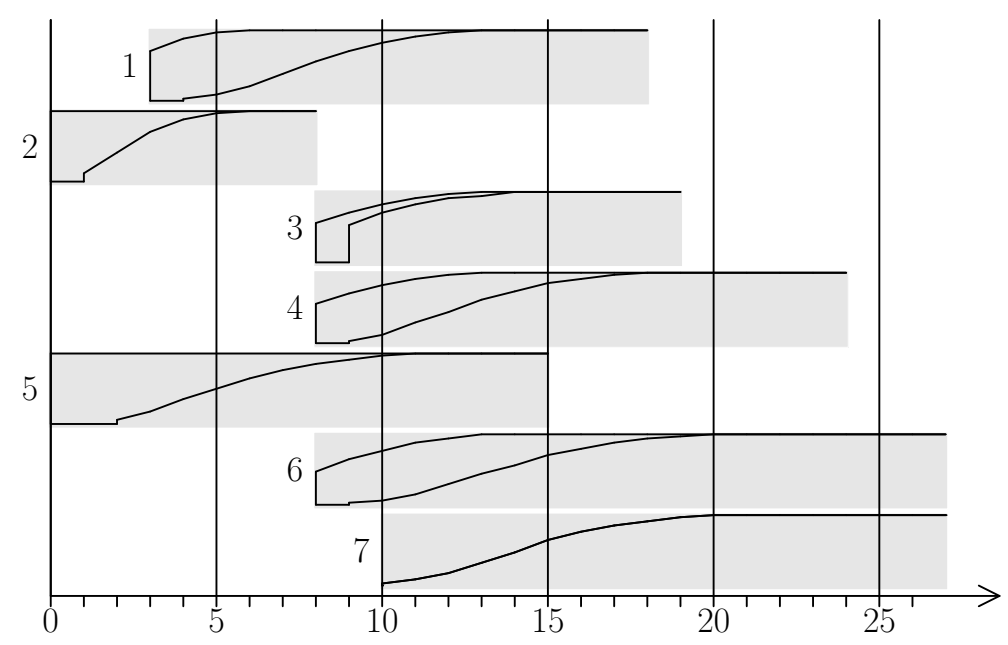

(a) $\Pi_{0}$ policy

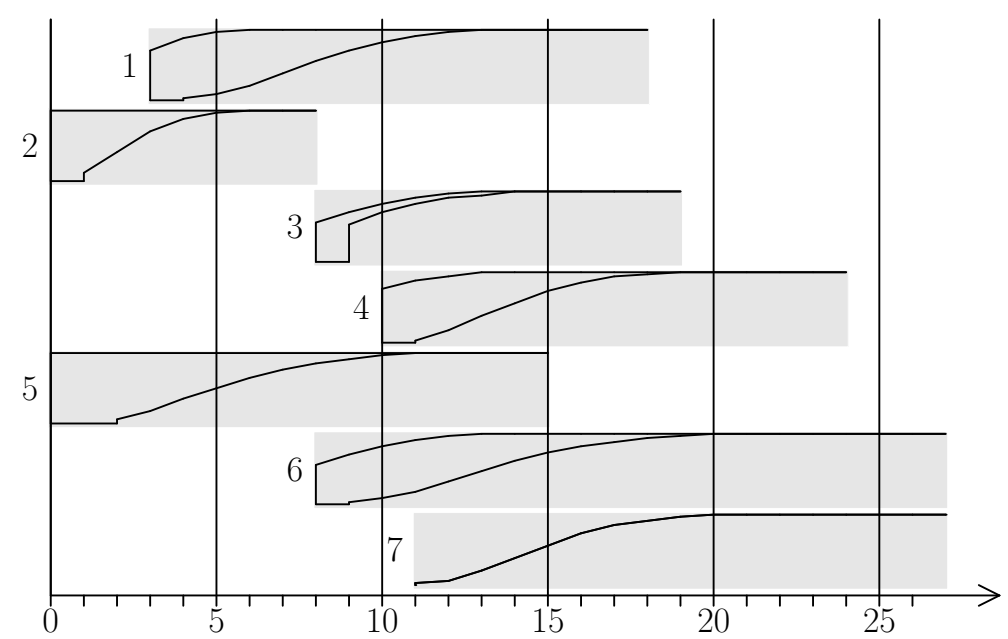

(b) STC + D policy

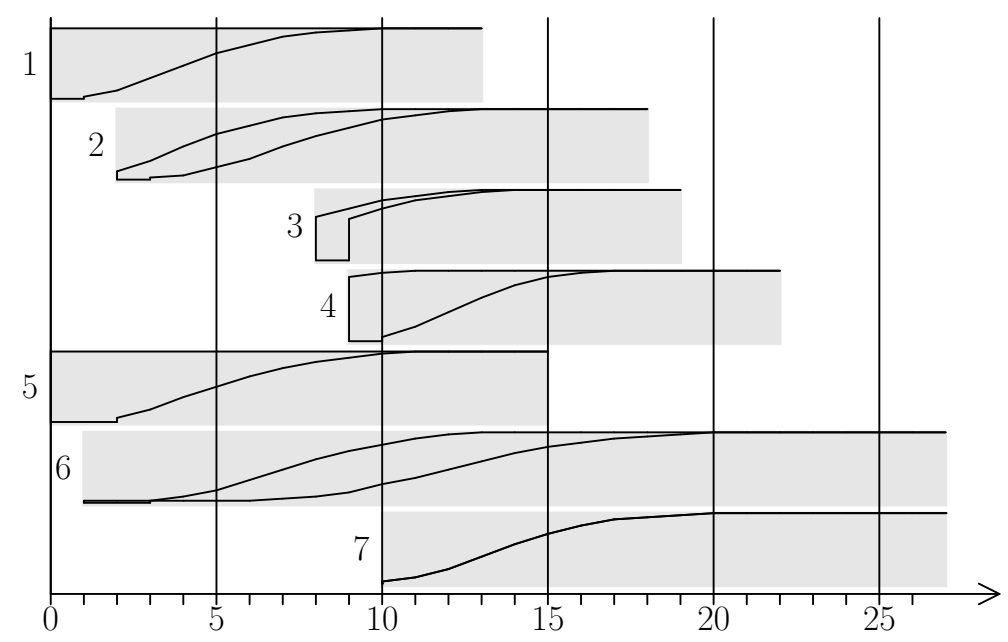

(c) Algorithm 2 policy

Figure 3: Stochastic Gantt charts 


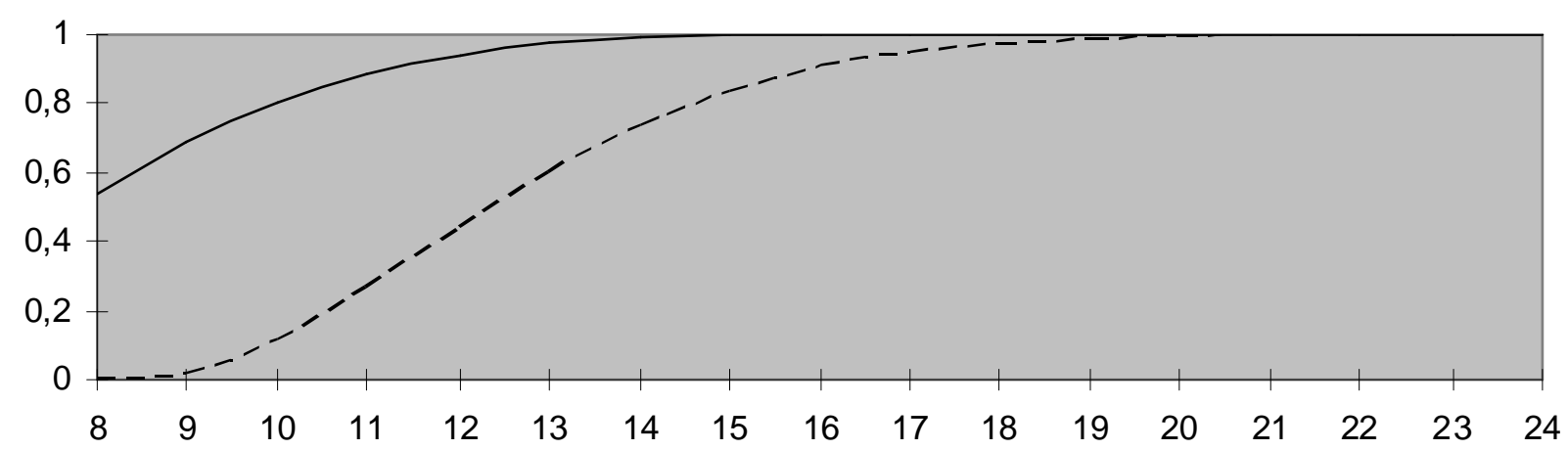

Figure 4: Stochastic Gantt chart (detail)

the start time of an activity. The right edge represents the maximum value for the finish time of the activity. The top edge of a grey bar corresponds to a cumulative probability equal to one, while the bottom edge corresponds to a cumulative probability equal to zero. The activity number is shown to the left of the corresponding horizontal bar. Note that activity 7 is the dummy end activity, and its (start or finish time) distribution is actually the project makespan distribution.

We can now visually compare the three different policies. The stochastic Gantt chart corresponding to the STC+D policy is shown in Figure 3 (b). When we compare this policy to $\Pi_{0}$ (see Figure 3 (a)), we see that not much has changed. The variability of $\boldsymbol{s}_{1}$ remains the same while the variability of $\boldsymbol{s}_{4}$ is reduced to the detriment of the makespan performance. Nevertheless, the increase of the expected penalty due to the violation of the due date is smaller than the reduction of the expected costs with respect to deviations from $\mathfrak{s}_{4}$, such that the total costs are reduced. This can also be verified in Figure 2 . The visualization of the Algorithm 2 policy shows a different picture (see Figure 3 (c)). The algorithm has shifted activity 1 to the very start of the project, eliminating all variation (and associated costs) of $\boldsymbol{s}_{1}$. This results in a high variability of $\boldsymbol{s}_{2}$, which is no problem since activity 2 is characterized as flexible. This beneficial switch has as a second consequence that the variability in $\boldsymbol{s}_{4}$ is easier to control, while the STC+D policy suffers from the precedence constraint $1 \prec 4$. Finally, the Algorithm 2 policy allows more variability in the starting time of the flexible activity 6 , which is beneficial for the makespan performance. The $\mathrm{STC}+\mathrm{D}$ procedure cannot make a similar decision, because activities 1 and 6 constitute a forbidden set, forcing the release time $s_{6}^{\text {buf }}$ to be greater than or equal to $s_{1}^{\text {buf }}+E\left(\boldsymbol{d}_{1}\right)$. For a similar reason, the $\mathrm{STC}+\mathrm{D}$ procedure is unable to schedule activity 1 before activity 2 .

This example shows that by making the policy generation procedure independent from deterministic schedules or resource constraints, Algorithm 2 is able to generate execution policies that are superior to those generated by the $\mathrm{STC}+\mathrm{D}$ procedure in terms of the policy execution cost (5).

\section{Computational results}

\subsection{Computational setup}

In this section we will verify whether the nice result of the example in the previous section can be generalized. For our computational experiments we used the well-known J30, J60 and J120 instance sets of PSPLIB (Kolisch and Sprecher, 1997). Both the STC+D 


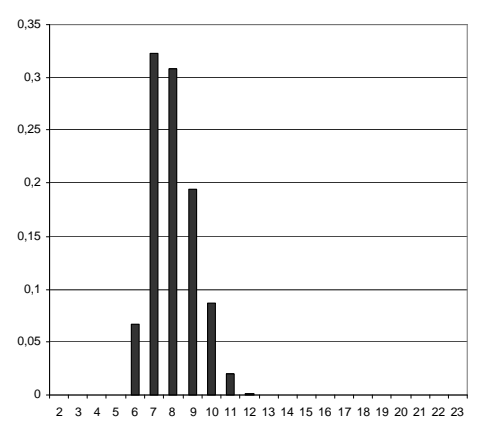

(a) Low variability

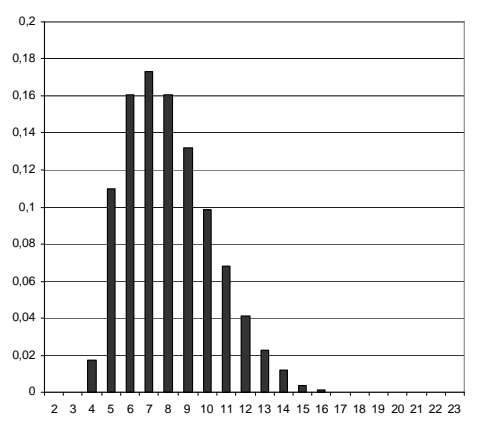

(b) Medium variability

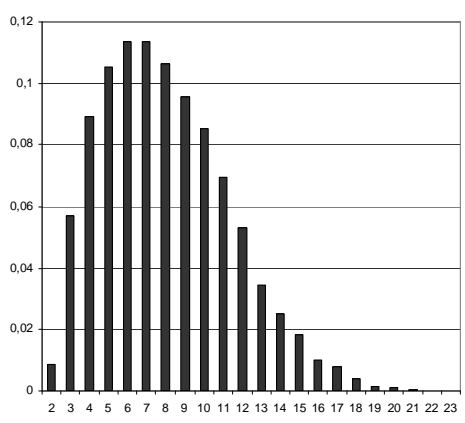

(c) High variability

Figure 5: Three levels of activity duration variability

procedure and Algorithm 2 require a deterministic RCPSP schedule as part of the input. For the problems of the J30 instance set, we have used the makespan minimizing branchand-bound algorithm of Demeulemeester and Herroelen $(1992,1997)$ for that purpose. Heuristic schedules for the J60 and J120 instance sets have been obtained by the combined crossover algorithm by Debels and Vanhoucke (2006) using 25,000 schedule generations as a stopping criterion. For every activity $i$, we generated a discretized beta distribution with shape parameters 2 and 5 , and an expected value $E\left(\boldsymbol{d}_{i}\right)$ equal to the deterministic PSPLIB activity duration. The activity duration distribution has either a small, medium or large variability, all with equal probability. In the case of a small variability, the minimum and maximum values of the stochastic variable $\boldsymbol{d}_{i}$ are equal to $0.75 * E\left(\boldsymbol{d}_{i}\right)$ and $1.625 * E\left(\boldsymbol{d}_{i}\right)$, respectively. In the case of a medium and a large variability, the corresponding intervals are $\left[0.5 * E\left(\boldsymbol{d}_{i}\right), 2.25 * E\left(\boldsymbol{d}_{i}\right)\right]$ and $\left[0.25 * E\left(\boldsymbol{d}_{i}\right), 2.875 * E\left(\boldsymbol{d}_{i}\right)\right]$, respectively. The probability distributions corresponding to the three possible variability levels are illustrated in Figure 5 for an activity with $E(\boldsymbol{d})_{i}=8$.

Every nondummy activity $i$ has a $50 \%$ chance of being inflexible. In that case, the $\operatorname{costs} c_{i}^{+}$and $c_{i}^{-}$are drawn from a discrete triangular distribution with $P\left(c_{i}^{+}=q\right)=$ $P\left(c_{i}^{-}=q\right)=(21-2 q) \%$ for $q \in\{1,2 \ldots .10\}$ (this is the same distribution as used in Van de Vonder et al. (2008)). This distribution results in a higher occurrence probability for low costs and in an average cost $c_{\text {avg }}=3.85$. Flexible activities have $c_{i}^{+}=c_{i}^{-}=0$. All algorithms have been coded in Visual $\mathrm{C}++$, and the results have been obtained on a personal computer equipped with an Intel ${ }^{\circledR}$ Xeon ${ }^{\circledR} 2.33 \mathrm{GHz}$ processor.

\subsection{Performance of the algorithm}

In a first set of experiments, we compare the performance of Algorithm 2 to the STC+D procedure. Recall that the $\mathrm{STC}+\mathrm{D}$ procedure determines a vector of starting times $s_{i}^{\text {buf }}$ that act as both release times and predictive starting times. Because the STC+D procedure was developed for a less general problem setting than the one described in Section 2, we first restrict ourselves to the problem setting proposed in Van de Vonder et al. (2008). More precisely, we assume symmetric costs, no bonus for the early completion of the project and a marginal cost $c_{n+1}=\left\lfloor 10 c_{\text {avg }}\right\rfloor=38$ per time unit violation of the project due date $\delta$. Also, contrary to what is proposed in Van de Vonder et al. (2008), we do not calculate the objective function (6), but use the following alternative formula instead:

$$
\tilde{\mathcal{C}}=\sum_{i=1}^{n} c_{i} E\left|\mathfrak{s}_{i}-\boldsymbol{s}_{i}\right|+c_{n+1} E\left(\max \left(0,\left(\boldsymbol{s}_{n+1}-\delta\right)\right)\right)
$$




\begin{tabular}{|c|c|c|c|}
\hline & J30 & J60 & J120 \\
\hline $1.01 \cdot E\left(C_{\max }\right)$ & $18.7 \%$ & $22.7 \%$ & $32.8 \%$ \\
$1.05 \cdot E\left(C_{\max }\right)$ & $19.9 \%$ & $24.1 \%$ & $34.7 \%$ \\
$1.10 \cdot E\left(C_{\max }\right)$ & $21.0 \%$ & $25.4 \%$ & $35.2 \%$ \\
\hline
\end{tabular}

Table 3: Improvement of Algorithm 2 over STC $+\mathrm{D}$

Since we use the optimal predictive starting times $\mathfrak{s}_{i}$ instead of the release times $s_{i}^{\text {buf }}$, we have $\tilde{\mathcal{C}} \leq \mathcal{C}^{\prime}$. Using Equation (10) instead of (6) for the evaluation of the STC+D procedure allows for a more fair comparison, as both STC $+\mathrm{D}$ and Algorithm 2 then use Algorithm 1 for the determination of the optimal predictive starting times.

We have tested the STC+D procedure and Algorithm 2 on the J30, J60 and J120 sets of PSPLIB, using a due date $\delta$ equal to $1.01 \cdot E\left(C_{\max }\right), 1.05 \cdot E\left(C_{\max }\right)$ and $1.1 \cdot E\left(C_{\max }\right)$, where $E\left(C_{\max }\right)$ is calculated as the expected makespan when we execute the project using the policy $\Pi_{0}$. Recall that $\Pi_{0}$ represents a rather competitive policy in terms of expected makespan (see Section 4.2). The higher the value of $\delta$, the more "room" the procedures will have to improve the objective function. A low value of $\delta$ implies that makespan performance is more important than the protection of inflexible activities. In Table 3, we show the average relative improvement of Algorithm 2 over the STC+D procedure, calculated as $\frac{\tilde{\mathcal{C}}(\mathrm{STC}+\mathrm{D})-\tilde{\mathcal{C}} \text { (Algorithm 2) }}{\tilde{\mathcal{C}}(\mathrm{STC}+\mathrm{D})} \cdot 100 \%$. We see that the policies generated by Algorithm 2 are substantially better than the ones generated by the STC $+\mathrm{D}$ procedure. The differences become bigger when the number of activities and/or the due date increases.

We have also evaluated both procedures in a more general problem setting. We now allow the costs to be asymmetric (i.e. for a given activity $i$ the deviation $\operatorname{costs} c_{i}^{+}$and $c_{i}^{-}$are independent draws from the triangular distribution described in the previous section) and we assume the presence of an earliness bonus $c_{n+1}^{-}=-\left\lfloor 5 c_{\text {avg }}\right\rfloor=-19$. The earliness bonus is (in absolute value) half as large as the tardiness penalty. We want to stress that the $\mathrm{STC}+\mathrm{D}$ procedure is disadvantaged here because it was not designed to work with asymmetric costs or bonuses for early project completion. The results of this experiment are shown in Table 4. The important observation here is that the differences in performance between the two algorithms can grow very large if the earliness costs or bonuses are ignored as is the case with the $\mathrm{STC}+\mathrm{D}$ algorithm. As before, the relative performance of Algorithm 2 increases as the due date increases. The reader may notice a seemingly strange trend in the results when looking at different project network sizes for a given due date. This is because two opposing forces are in effect. On the one hand, there is the fact that Algorithm 2 has a better relative performance than the STC+D procedure as the number of activities increases (see the results in Table 3). On the other hand, when the number of (nondummy) activities increases, the relative importance (in the objective function) of bonuses/penalties related to the due date decreases. In other words, when the number of nondummy activities increases, it makes the "handicap" of the STC $+\mathrm{D}$ procedure (ignoring earliness bonuses) less severe. Some of the percentages in Table 4 are larger than $100 \%$ because the corresponding policy execution costs have negative values.

The average computation times (in seconds) of the second experiment are shown in Table 5. The computation times of Algorithm 2 are substantially higher than the computation times of the $\mathrm{STC}+\mathrm{D}$ procedure. This is no surprise, because all the components of Algorithm 2 rely on simulation to evaluate the objective function. For the largest instance set, the average computation time lies around 25 minutes, which is still acceptable. 


\begin{tabular}{|c|r|r|r|}
\hline & \multicolumn{1}{|c|}{$\mathrm{J} 30$} & \multicolumn{1}{|c|}{$\mathrm{J} 60$} & \multicolumn{1}{|c|}{$\mathrm{J} 120$} \\
\hline $1.01 \cdot E\left(C_{\max }\right)$ & $37 \%$ & $32 \%$ & $33 \%$ \\
$1.05 \cdot E\left(C_{\max }\right)$ & $104 \%$ & $67 \%$ & $53 \%$ \\
$1.10 \cdot E\left(C_{\max }\right)$ & $166 \%$ & $515 \%$ & $378 \%$ \\
\hline
\end{tabular}

Table 4: Improvement of Algorithm 2 over STC $+\mathrm{D}$ in a general problem setting

(a) Computation times of Algorithm 2

\begin{tabular}{|c|c|c|c|}
\hline & $\mathrm{J} 30$ & $\mathrm{~J} 60$ & $\mathrm{~J} 120$ \\
\hline $1.01 \cdot E\left(C_{\max }\right)$ & 8.4 & 94 & 1631 \\
$1.05 \cdot E\left(C_{\max }\right)$ & 7.8 & 84 & 1475 \\
$1.10 \cdot E\left(C_{\max }\right)$ & 7.1 & 77 & 1371 \\
\hline
\end{tabular}

(b) Computation times of STC $+\mathrm{D}$

\begin{tabular}{|l|l|l|l|}
\hline & $\mathrm{J} 30$ & $\mathrm{~J} 60$ & $\mathrm{~J} 120$ \\
\hline $1.01 \cdot E\left(C_{\max }\right)$ & 0.1 & 1.4 & 12.9 \\
$1.05 \cdot E\left(C_{\max }\right)$ & 0.1 & 1.7 & 14.8 \\
$1.10 \cdot E\left(C_{\max }\right)$ & 0.2 & 2.3 & 19.1 \\
\hline
\end{tabular}

Table 5: Computation times

Furthermore, the imposed due date does not have a very significant effect on the required computation time of Algorithm 2. The reader will notice that the computation times of Algorithm 2 seem to decrease with increasing due date, while the opposite holds for the $\mathrm{STC}+\mathrm{D}$ procedure. For Algorithm 2, the computation time decrease can be attributed to a decrease in the computation time of the improvement procedure (Algorithm 4). For the STC $+\mathrm{D}$ procedure, the computation time increase can be largely attributed to the descent part of the procedure and to a lesser extent to the STC part of the procedure.

The small increase in computation times of Algorithm 2 when the due date decreases is because during the initial policy generation procedure (Algorithm 3) moves only result in release time increases. When we remove the resource constraints during the improvement procedure (Algorithm 4), a lot of release time decreases will result in an improvement of the objective function, because of the small due date. This results in more moves being made in total, and hence a slightly larger computation time. The computation time increase of the $\mathrm{STC}+\mathrm{D}$ procedure with increasing due date, is because the descent procedure in STC+D only considers resource feasible starting times. When the due date is small, the schedule will have little slack, and only a small number of moves must be considered. A larger due date on the other hand implies more slack in the schedule and thus a greater number of moves to be evaluated. Hence the larger computation time. In Algorithm 4, there is no such thing as slack, and as a consequence that variable does not influence the running time of the algorithm.

\subsection{Performance of the improvement procedures}

In this section, we study the impact of the different improvement procedures of Algorithm 2. To do this, we omit one of the algorithm components and compare the performance of the resulting procedure to the performance of the original procedure. We used the experimental setup of the second experiment described in the previous section, and conducted the experiment on the J60 instance set with a due date equal to $1.05 \cdot E\left(C_{\text {max }}\right)$. The results are shown in Table 6 . We see that the improvement procedure itself (Algorithm 4) results in a very significant improvement of the solution generated by the initial policy generation procedure (Algorithm 3). The three parts of Algorithm 4 have a more or less equal contribution to this improvement, but there are clearly large interaction effects since the three percentages (by far) do not sum up to $273 \%$. 


\begin{tabular}{|l|c|}
\hline & $\begin{array}{c}\text { Performance } \\
\text { decrease }\end{array}$ \\
\hline Without improvement procedure (Algorithm 4) & $273 \%$ \\
Without broad neighborhood search on $\vec{\tau}$ (Algorithm 5) & $41 \%$ \\
Without neighborhood search on $\vec{\pi}$ (Algorithm 6) & $31 \%$ \\
Without narrow neighborhood search on $\vec{\tau}$ (Algorithm 7) & $41 \%$ \\
\hline
\end{tabular}

Table 6: Performance of the improvement procedures

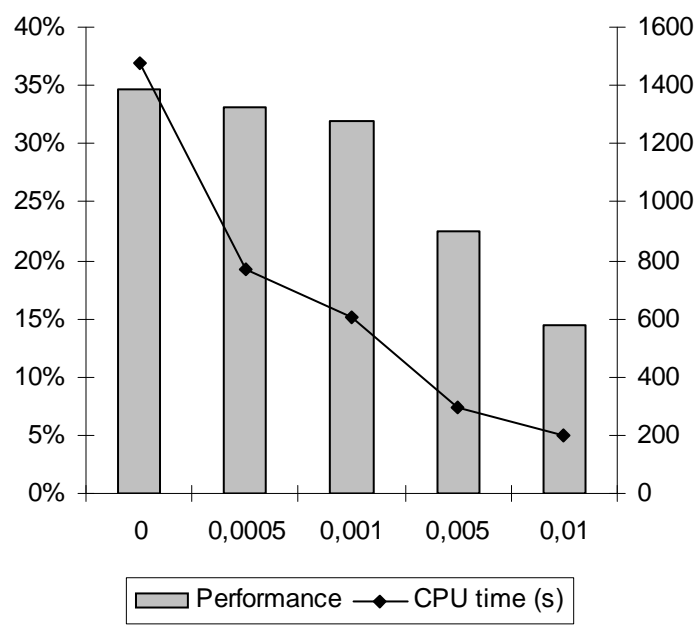

(a) Algorithm performance

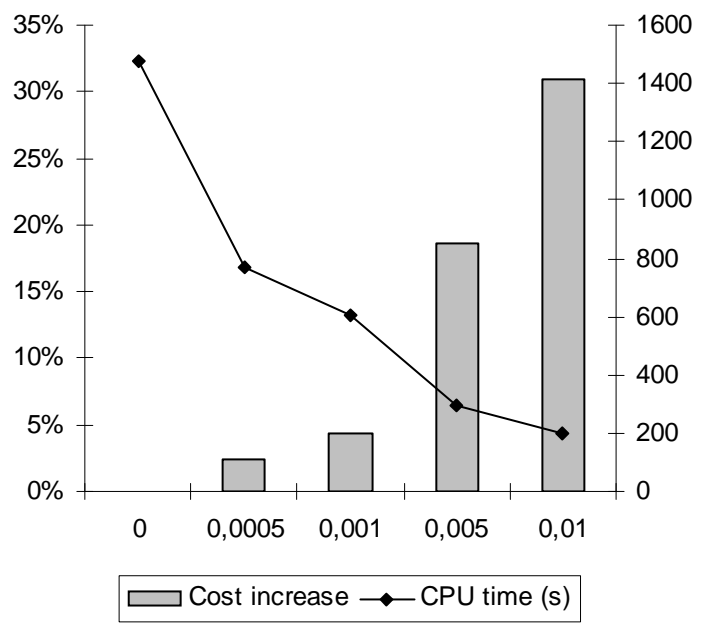

(b) Cost increase

Figure 6: Algorithm performance for different values of $\epsilon$

\subsection{Speeding up the algorithm}

Since the computation times of Algorithm 2 are rather high for large instances, we will investigate an opportunity to speed up the algorithm, at the cost of the solution quality. A very straightforward way to speed up Algorithm 2 is as follows. Whenever we are about to execute an improving move (during any part of Algorithm 2), we can choose to execute it only if the relative policy execution cost decrease is higher than a certain threshold value $\epsilon$. Formally, given the objective value of the current solution $\mathcal{C}_{\text {old }}$ and a move that is about to be executed that would result in a new objective value $\mathcal{C}_{\text {new }}<\mathcal{C}_{\text {old }}$, we execute the move only if $\frac{\mathcal{C}_{\text {old }}-\mathcal{C}_{\text {new }}}{\mathcal{C}_{\text {old }}}>=\epsilon$. Clearly, if we set $\epsilon=\infty$, the algorithm will stop very quickly because no move will be executed. If we set $\epsilon=0$, the algorithm will exhibit the same speed and performance as before. We are interested in the results obtained when using a value in between those two extremes.

In order to evaluate the impact of different values for $\epsilon$, we have performed a computational experiment on the 120-activity instance set, with a due date equal to $1.05 \cdot E\left(C_{\max }\right)$ and in the same problem setting as the results shown in Table 3. For different values of $\epsilon$, we have measured the average computation time, the performance of Algorithm 2 as the percentage improvement over STC+D, and the percentage performance decrease (w.r.t. the objective value) when compared to the case where $\epsilon=0$. The results are shown in Figure 6. The different datapoints in Figures 6 (a) and (b) correspond to different values of $\epsilon$ (indicated below the $X$-axis). The bars correspond to the algorithm performance (with respect to the objective value) and are drawn relative to the left $Y$-axis, while the solid line corresponds to the CPU time (in seconds) and should be interpreted relative 
to the right $Y$-axis. The difference between the two panes of Figure 6 is the way the algorithm performance is measured.

In Figure 6 (a), we show the algorithm performance as the percentage improvement over the STC $+\mathrm{D}$ procedure. For $\epsilon=0$, we obtain the original result of $34.7 \%$. As $\epsilon$ increases, the algorithm performance decreases along with the CPU time, as was expected. We see that for all the tested values of $\epsilon$, Algorithm 2 still outperforms the STC+D procedure, but the performance significantly degrades as $\epsilon$ increases. In Figure 6 (b), the algorithm performance is measured as the percentage deterioration of the objective function compared to the case where $\epsilon=0$. A nice result is obtained for $\epsilon=0.0005$ : the resulting average policy execution cost is only $2.5 \%$ higher than the "original" value, but the algorithm runs twice as fast. For large instances, this may constitute an attractive trade-off between computation time and solution quality. If we go beyond this threshold of 0.0005 , however, more significant speedups inevitably result in solutions of substantially lower quality.

Finally, we note that we have also tried to speed up the algorithm by simply reducing the number of scenarios used for the evaluation of the objective function, but we found that this measure was less attractive in the sense that for an equally large speedup, a significantly greater performance loss was obtained when compared to the method described above.

\section{Conclusions}

In this paper, we studied the problem of the execution of a resource-constrained project when activity durations are uncertain. We formulated a problem where one tries to determine a project execution policy and a vector of predictive activity starting times such that the policy execution costs are minimized. These policy execution costs consist of costs related to positive and negative deviations from the predictive activity starting times of inflexible activities, as well as the costs associated with exceeding the project due date and the bonus opportunities related to early project completion. We showed that this problem setting is more general than related problem descriptions found in the literature.

For this general problem setting, we have proposed a solution procedure that is essentially a combination of four descent procedures that heavily rely on simulation for the evaluation of the objective function. The result of this procedure is a vector of release times and a priority list, that are to be used in a simple modified parallel schedule generation scheme. Given this execution policy, we proposed a methodology based on the newsvendor problem to derive the corresponding vector of optimal predictive starting times. We have graphically and numerically illustrated the benefits of our procedure on an example instance. In computational experiments, our procedure has been compared to the state-of-the-art STC+D procedure, and we found that our procedure is vastly superior in performance, at the cost of a larger average computation time. Finally, we have proposed a way to speed up our procedure, with the interesting result that the computation time can be divided by two without sacrificing much of the solution quality. 


\section{References}

Al-Turki, U. M., Mittenthal, J. and Raghavachari, M. (1996). The single-machine absolute-deviation early-tardy problem with random completion times. Naval Research Logistics, 43(4), pp 573-587.

Arrow, K., Harris, T. and Marshack, J. (1951). Optimal inventory policy. Econometrica, 19 , pp 250-272.

Artigues, C., Michelon, P. and Reusser, S. (2003). Insertion techniques for static and dynamic resource-constrained project scheduling. European Journal of Operational Research, 149(2), pp 249-267.

Ashtiani, B., Leus, R. and Aryanezhad, M. (2009). New competitive results for the stochastic resource-constrained project scheduling problem: exploring the benefits of pre-processing. European Journal of Operational Research, to appear.

Baker, K. and Trietsch, D. (2009). Principles of sequencing and scheduling. Wiley, Hoboken, New Jersey.

Ballestín, F. and Leus, R. (2009). Resource-constrained project scheduling for timely project completion with stochastic activity durations. Production and Operations Management, 18, pp 459-474.

Brooks, G. and White, C. (1965). An algorithm for finding optimal or near optimal solutions to the production scheduling problem. Journal of Industrial Engineering, 16, pp 34-40.

Debels, D. and Vanhoucke, M. (2006). Future research avenues for resource constrained project scheduling: Search space restriction or neighbourhood search extension. Research report. Ghent University, Belgium.

Debels, D. and Vanhoucke, M. (2007). A decomposition-based genetic algorithm for the resource-constrained project scheduling problem. Operations Research, 55(3), pp 457469.

Deblaere, F., Demeulemeester, E. and Herroelen, W. (2010). Reactive scheduling in the multi-mode rcpsp. Computers and Operations Research, to appear.

Demeulemeester, E. and Herroelen, W. (1992). A branch-and-bound procedure for the multiple resource-constrained project scheduling problem. Management Science, 38, pp 1803-1818.

Demeulemeester, E. and Herroelen, W. (1997). New benchmark results for the resourceconstrained project scheduling problem. Management Science, 43, pp 1485-1492.

Hagstrom, J. (1988). Computational complexity of PERT problems. Computers and Operations Research, 18, pp 139-147.

Herroelen, W., De Reyck, B. and Demeulemeester, E. (2000). On the paper "Resourceconstrained project scheduling: Notation, classification, models and methods" by Brucker et al.. European Journal of Operational Research, 128(3), pp 221-230. 
Kolisch, R. and Sprecher, A. (1997). PSPLIB - A project scheduling library. European Journal of Operational Research, 96, pp 205-216.

Lambrechts, O. (2007). Robust project scheduling subject to resource breakdowns. PhD thesis. Department of Decision Sciences and Information Management (KBI), K.U. Leuven.

Lambrechts, O., Demeulemeester, E. and Herroelen, W. (2007). A tabu search procedure for developing robust predictive project schedules. International Journal of Production Economics, 111(2), pp 496-508.

Lambrechts, O., Demeulemeester, E. and Herroelen, W. (2008). Proactive and reactive strategies for resource-constrained project scheduling with uncertain resource availabilities. Journal of Scheduling, 11(2), pp 121-136.

Leus, R. (2003). The generation of stable project plans. PhD thesis. Department of applied economics, Katholieke Universiteit Leuven, Belgium.

Leus, R. and Herroelen, W. (2004). Stability and resource allocation in project planning. IIE Transactions, 36(7), pp 1-16.

Möhring, R., Radermacher, F. and Weiss, G. (1984). Stochastic scheduling problems I Set strategies. Zeitschrift für Operations Research, 28, pp 193-260.

Möhring, R., Radermacher, F. and Weiss, G. (1985). Stochastic scheduling problems II General strategies. Zeitschrift für Operations Research, 29, pp 65-104.

Schatteman, D., Herroelen, W., Van de Vonder, S. and Boone, A. (2008). A methodology for integrated risk management and proactive scheduling of construction projects. Journal of Construction Engineering and Management, 134(11), pp 885-893.

Stork, F. (2001). Stochastic resource-constrained project scheduling. PhD thesis. Technical University of Berlin, School of Mathematics and Natural Sciences.

Tian, W. and Demeulemeester, E. (2010). Railway scheduling reduces the expected project makespan. Research Report 1004. Department of Decision Sciences and Information Management, K.U.Leuven, Belgium, 32 pp.

Van de Vonder, S. (2006). Proactive-reactive procedures for robust project scheduling. $\mathrm{PhD}$ thesis. Department of Decision Sciences and Information Management (KBI), K.U. Leuven.

Van de Vonder, S., Ballestín, F., Demeulemeester, E. and Herroelen, W. (2007). Heuristic procedures for reactive project scheduling. Computers \& Industrial Engineering, $52(1)$, pp 11-28.

Van de Vonder, S., Demeulemeester, E. and Herroelen, W. (2008). Proactive heuristic procedures for robust project scheduling: An experimental analysis. European Journal of Operational Research, 189(3), pp 723-733.

Wasan, M. (1970). Parametric estimation. McGraw-Hill, New York.

Yu, G. and Qi, X. (2004). Disruption management - Framework, models and applications. World Scientific, New Jersey. 
Zhu, G., Bard, J. and Yu, G. (2005). Disruption management for resource-constrained project scheduling. Journal of the Operational Research Society, 56, pp 365-381. 